\title{
Section problems for configurations of points on the Riemann sphere
}

\author{
LEI CHEN \\ NiCK SALTER
}

\begin{abstract}
We prove a suite of results concerning the problem of adding $m$ distinct new points to a configuration of $n$ distinct points on the Riemann sphere, such that the new points depend continuously on the old. Altogether, these results provide a complete answer to the following question: given $n \neq 5$, for which $m$ can one continuously add $m$ points to a configuration of $n$ points? For $n \geq 6$, we find that $m$ must be divisible by $n(n-1)(n-2)$, and we provide a construction based on the idea of cabling of braids. For $n=3,4$, we give some exceptional constructions based on the theory of elliptic curves.
\end{abstract}

20F36, 55S40

\section{Introduction}

This paper studies the space $\operatorname{Conf}_{n}\left(S^{2}\right)$ of configurations of $n$ distinct unordered points in $S^{2}$. This is the base space for a fiber bundle $P: \operatorname{Conf}_{n, m}\left(S^{2}\right) \rightarrow \operatorname{Conf}_{n}\left(S^{2}\right)$, where the total space $\operatorname{Conf}_{n, m}\left(S^{2}\right)$ is the space of configurations of $n+m$ distinct points divided into two groups of cardinalities $n$ and $m$. For any fiber bundle $\pi: E \rightarrow B$, it is a basic question to understand the space of sections, ie continuous maps $\sigma: B \rightarrow E$ satisfying $\pi \circ \sigma=\mathrm{id}$. In the case of $P: \operatorname{Conf}_{n, m}\left(S^{2}\right) \rightarrow \operatorname{Conf}_{n}\left(S^{2}\right)$, a section $S: \operatorname{Conf}_{n}\left(S^{2}\right) \rightarrow \operatorname{Conf}_{n, m}\left(S^{2}\right)$ has a very natural interpretation: $S$ is an assignment of $m$ additional distinct points to a given configuration of $n$ distinct points that depends continuously on the position of the $n$ points.

The approach we pursue in this paper is to study sections of $P$ by means of the fundamental group. The spherical braid group $B_{n}\left(S^{2}\right)$ is the fundamental group of $\operatorname{Conf}_{n}\left(S^{2}\right)$, and we also define

$$
B_{n, m}\left(S^{2}\right):=\pi_{1}\left(\operatorname{Conf}_{n, m}\left(S^{2}\right)\right) .
$$


Setting $p:=P_{*}$ and $s:=S_{*}$, a section $S$ induces a group-theoretic section

$$
s: B_{n}\left(S^{2}\right) \rightarrow B_{n, m}\left(S^{2}\right)
$$

of the surjective homomorphism $p: B_{n, m}\left(S^{2}\right) \rightarrow B_{n}\left(S^{2}\right)$. Thus an obstruction to the existence of a group-theoretic section $s$ furnishes an obstruction to the existence of a bundle-theoretic section $S$. A standard argument in obstruction theory shows that the converse is true as well.

The theory of sections of bundles of configuration and moduli spaces plays an important role in topology, geometric group theory, and algebraic geometry. See, for instance, the classic papers of Earle-Kra [6; 7] and Hubbard [13], the work of Lin [14], or the recent work of W Chen [4], each of which treats various instances of the problem of obstructing and classifying sections of such bundles.

The section problem for $P: \operatorname{Conf}_{n, m}\left(S^{2}\right) \rightarrow \operatorname{Conf}_{n}\left(S^{2}\right)$ is particularly subtle and rich for several reasons. If the ambient space $S^{2}$ is replaced with $\mathbb{C}$, sections of $\operatorname{Conf}_{n, m}(\mathbb{C}) \rightarrow \operatorname{Conf}_{n}(\mathbb{C})$ are easy to construct: one can simply add $m$ new points "near infinity". By contrast, even the mere existence of sections in the spherical case is far from obvious. Our interest in this question was sparked by a 2005 paper of Gonçalves-Guaschi [10]. They established the following pioneering and intriguing theorem.

Theorem 1.1 (Gonçalves-Guaschi) A group-theoretic section $s: B_{3}\left(S^{2}\right) \rightarrow B_{3, m}\left(S^{2}\right)$ exists if and only if $m \equiv 0$ or $m \equiv 2(\bmod 3)$.

For $n \geq 4$, there are no sections $s: B_{n}\left(S^{2}\right) \rightarrow B_{n, m}\left(S^{2}\right)$ except possibly if $m$ is congruent to one of the four residues

$0, \quad(n-1)(n-2), \quad-n(n-2), \quad-(n-2) \quad \bmod n(n-1)(n-2)$.

See below for a more complete discussion of the work of Gonçalves-Guaschi and the role that their work plays in informing the present approach.

Main results: statement Gonçalves-Guaschi did not give any explicit construction of sections, even in the $n=3$ case. The results of this paper complete the analysis begun in Theorem 1.1 by finding further obstructions to sections unseen by the methods of Gonçalves-Guaschi (Theorem A) and by providing explicit constructions of sections in all but one of the cases not obstructed by Theorem A; these results appear as Theorems B and C. Taken together, the results of the paper give a complete determination of those $m$ for which there exist sections $S: \operatorname{Conf}_{n}\left(S^{2}\right) \rightarrow \operatorname{Conf}_{n, m}\left(S^{2}\right)$, excepting $n=5$. 
Theorem A (obstruction of sections) No section $S: \operatorname{Conf}_{n}\left(S^{2}\right) \rightarrow \operatorname{Conf}_{n, m}\left(S^{2}\right)$ exists for $n \geq 6$ unless $n(n-1)(n-2)$ divides $m$.

We find that for arbitrary $n$, one can build a family of sections using the idea of "cabling" of braids.

Theorem B (cabling spherical braids) For any $n \geq 3$ and for any $m$ divisible by $n(n-1)(n-2)$, there is a section $S: \operatorname{Conf}_{n}\left(S^{2}\right) \rightarrow \operatorname{Conf}_{n, m}\left(S^{2}\right)$.

The exceptional sections for $n \leq 4$ are algebrogeometric in nature, and we switch from viewing $S^{2}$ as the 2 -sphere to the projective space $\mathbb{C P}^{1}$. Note that for $n=4$, the residues appearing in Theorem 1.1 are $0,6,16,22 \bmod 24$.

Theorem C (exceptional algebrogeometric sections) For any $m \geq 0$ satisfying $m \equiv 0,2 \bmod 3$, there exists an algebraic map $S$ that gives a section of the bundle $\operatorname{Conf}_{3, m}\left(\mathbb{C P}^{1}\right) \rightarrow \operatorname{Conf}_{3}\left(\mathbb{C P}^{1}\right)$.

There is a section $S: \operatorname{Conf}_{4}\left(\mathbb{C P}^{1}\right) \rightarrow \operatorname{Conf}_{4, m}\left(\mathbb{C P}^{1}\right)$ for any $m \geq 0$ congruent to one of $0,6,16,22 \bmod 24$.

Remark 1.2 The section problem in the cases $n=1,2$ reduces to some pleasant exercises in basic algebraic topology. For $n=1$, a section $s: \operatorname{Conf}_{1}\left(S^{2}\right) \rightarrow \operatorname{Conf}_{1,1}\left(S^{2}\right)$ is provided by the antipodal map, and indeed the Lefschetz fixed-point theorem and the Hopf degree theorem combine to show that this is the only such section up to homotopy. We claim that no section exists for $n=1$ and $m \geq 2$. Indeed, as $\operatorname{Conf}_{1}\left(S^{2}\right)=S^{2}$ is simply connected, the monodromy of any such section is trivial, and hence the points $s(z)_{1}, \ldots, s(z)_{m}$ associated to $z \in S^{2}$ by any putative $m$-point section would be globally ordered. If $m \geq 2$, one can use the unique Möbius transformation taking the ordered tuple $\left(z, s(z)_{1}, s(z)_{2}\right)$ to $(0,1, \infty)$ to give a framing of $S^{2}$, but no such framing exists. A similar analysis shows that no sections exist for $n=2$ and arbitrary $m \geq 1$.

In the remainder of the paper we install the standing assumption that $n \geq 3$; further restrictions on $n$ will be specified where necessary.

Main results: discussion Below we offer some further discussion of the three main results. We begin with the constructive Theorems B and C. 
Theorem B: cabling and rational maps Theorem B, discussed in Section 3, constructs sections in the case $m \equiv 0 \bmod n(n-1)(n-2)$. Geometrically, the idea is to construct a section by adding a "halo" of new points nearby each original point. In light of the nontriviality of the tangent bundle of $S^{2}$, the challenge is to give a suitable framing of the tangent space at the original points.

We provide two points of view on the solution to this problem, one more topological and group-theoretical, and one more algebraic. On the topological side, we appeal to the theory of "cabling" of braids. These are a family of homomorphisms $B_{n} \rightarrow B_{k n}$ arising from the simple idea of viewing each strand of a braid as being built from a "cable" of $k$ smaller strands. The idea of cabling is not novel (see the references listed in Section 3), but to the authors' knowledge, the specific challenges involved in analyzing cabling in the setting of the spherical braid group do not appear elsewhere in the literature. These details are presented in Section 3.

Our second point of view on Theorem $\mathrm{B}$ is via the theory of rational maps on $\mathbb{C P}^{1}$. Given three ordered distinct points $A, B$ and $C$, there is a unique Möbius transformation $\phi \in \operatorname{PSL}(2, \mathbb{C})$ such that $\phi(0)=A, \phi(1)=B$ and $\phi(\infty)=C$. To such a triple, we (continuously) associate the point $D:=\phi(2)$. However, in the unordered case, different orderings of 3 points give different Möbius transformations. We solve this problem by multiplying suitable collections of Möbius transformations together, allowing us to continuously assign multiple points to an unordered configurations of $n$ points. The appearance of the peculiar number $n(n-1)(n-2)$ is very natural from this point of view, as $n(n-1)(n-2)$ is the number of ordered triples of $n$ points. See more about the construction in Remark 3.6.

Theorem C: exceptional sections via algebraic geometry Theorem $\mathrm{C}$, discussed in Section 4, studies the exceptional cases $n=3$ and $n=4$, using ideas borrowed from algebraic geometry to construct some special sections. For $n=3$, the key idea is again the theory of Möbius transformations, appearing this time in the guise of the cross-ratio. For $n=4$, we appeal to the theory of elliptic curves. A configuration of 4 unordered points on $\mathbb{C P}^{1}$ gives rise, via a 2 -sheeted branched covering, to an elliptic curve. The branch points correspond to the 2-torsion points, and the basic insight is to construct sections by assigning to a given 4 -tuple the images of the $k$-torsion points of the associated elliptic curve for $k>2$.

The work of Gonçalves-Guaschi The present work is directly inspired by the work of Gonçalves-Guaschi [10] mentioned above. In order to discuss our final main result 
(Theorem A), we must first recall the work of Gonçalves-Guaschi. Their fundamental insight is to exploit the structure of torsion subgroups of $B_{n}\left(S^{2}\right)$. They observe that any section $s: B_{n}\left(S^{2}\right) \rightarrow B_{n, m}\left(S^{2}\right)$, being an injective group homomorphism, must send torsion elements to torsion elements of the same order. Work of Murasugi [15] (see Section 2.4) classifies torsion in $B_{n}\left(S^{2}\right)$ and finds that all torsion elements must have order dividing one of the numbers $2 n, 2 n-2,2 n-4$; this is one point of origin for the somewhat mysterious number $n(n-1)(n-2)$ that appears in Theorems 1.1 and A. Some elementary number theory, based on Murasugi's classification and the principle that $s$ preserves torsion order, establishes a first constraint on $m$ given $n$. Gonçalves-Guaschi finish their argument via group-cohomological techniques, computing an obstruction class that is nonvanishing in the cases where their theorem applies. To accomplish this, they make use of a generating set for $B_{n}\left(S^{2}\right)$ that is particularly convenient for analyzing torsion.

Theorem A: obstructions via the mapping class group Our method of proof for Theorem A follows Gonçalves-Guaschi in studying $B_{n}\left(S^{2}\right)$ from the point of view of its torsion subgroups. Whereas the technical core of the argument of GonçalvesGuaschi is built around group cohomology, we take a different approach, exploiting the close relationship between $B_{n}\left(S^{2}\right)$ and the mapping class $\operatorname{group}^{\operatorname{Mod}_{n}}\left(S^{2}\right)$ of the $n$-punctured sphere. The analogous section problem for mapping class groups is amenable to the powerful theory of canonical reduction systems. We prove Theorem A by analyzing the canonical reduction systems for a generating set of $\operatorname{Mod}_{n}\left(S^{2}\right)$ induced from the generating set for $B_{n}\left(S^{2}\right)$ studied by Gonçalves-Guaschi.

As to the missing case $n=5$, we conjecture that Theorem A should apply here as well, and we do not expect to find any exceptional algebrogeometric sections. Some of the arguments internal to Theorem A require at least 6 points (see Remark 6.1), but we hope that some more clever refinements applicable to $n \geq 5$ can be found.

It is natural to ask the extent to which these results are "predicted" by algebraic geometry. One way of making this precise is to ask whether every section $s: \operatorname{Conf}_{n}\left(S^{2}\right) \rightarrow$ $\operatorname{Conf}_{n, m}\left(S^{2}\right)$ is homotopic to a map of varieties $s^{\prime}: \operatorname{Conf}_{n}\left(\mathbb{C P}^{1}\right) \rightarrow \operatorname{Conf}_{n, m}\left(\mathbb{C P}^{1}\right)$. Perhaps surprisingly, it turns out that for $n \geq 5$ the projection map $p: \operatorname{Conf}_{n, m}\left(\mathbb{C P}^{1}\right) \rightarrow$ $\operatorname{Conf}_{n}\left(\mathbb{C P}^{1}\right)$ has no section given by an algebraic map. This is an instance of a theorem of Lin [14, Theorem 3], which builds off of the work of Earle-Kra [6, Section 4.6]. Thus the problem of constructing and classifying continuous sections of $\operatorname{Conf}_{n, m}\left(S^{2}\right) \rightarrow$ $\operatorname{Conf}_{n}\left(S^{2}\right)$ is genuinely different from the analogous problem in the algebraic category. 
The results of this paper only concern the (non)existence of sections of $\operatorname{Conf}_{n, m}\left(S^{2}\right) \rightarrow$ $\operatorname{Conf}_{n}\left(S^{2}\right)$. We have not addressed the question of uniqueness, but we believe that this is worthy of further study.

Question 1.3 What is the homotopy type of the space of sections of $\operatorname{Conf}_{n, m}\left(S^{2}\right) \rightarrow$ $\operatorname{Conf}_{n}\left(S^{2}\right)$ ? Is every section $S: \operatorname{Conf}_{n}\left(S^{2}\right) \rightarrow \operatorname{Conf}_{n, m}\left(S^{2}\right)$ homotopic to one of the sections constructed in Theorem $B$ or Theorem $C$ ?

Contents of the paper Section 2 recalls some basic facts about the groups $B_{n}\left(S^{2}\right)$ and $\operatorname{Mod}_{n}\left(S^{2}\right)$. Section 3 discusses "cabling" of braids and exploits this to give the construction from which Theorem B follows. In Section 4, we give some algebrogeometric constructions of sections in the cases $n=3$, 4 , establishing Theorem $\mathrm{C}$.

The proof of Theorem A is carried out in Sections 5-10. In Section 5, we review the theory of canonical reduction systems. In Section 6 we establish some preliminary notions, leading to an overview of the proof given in Section 7. The proof itself is carried out in Sections 8-10.

Acknowledgements The authors would like to thank Ian Frankel for some helpful suggestions concerning Theorem C, and Dmitri Gekhtman for some insights in Teichmüller theory. Salter would like to thank Khanh Le for the observation that $22=6+16$, leading to a simplification and strengthening of Theorem $\mathrm{C}$. The authors are grateful to Joan Birman and to an anonymous referee for comments on preliminary drafts that improved the exposition.

Salter gratefully acknowledges support by the National Science Foundation under Award No. DMS-1703181.

\section{The spherical braid group}

In this section, we remind the reader of the relevant aspects of the theory of the spherical braid groups and their relationship with the mapping class groups of the punctured sphere. Most of the results in this section can be found in [11, Section 4].

\subsection{The (spherical) braid group}

Let $S$ be a surface of finite type and let $\operatorname{PConf}_{k}(S)$ denote the space of ordered $k$-tuples of distinct points on $S$. The symmetric group $S_{k}$ acts on $\operatorname{PConf}_{k}(S)$ by 
permuting the ordering of the points; this action is by deck transformations. For any subgroup $G \leq S_{k}$, there is an associated covering space $\operatorname{PConf}_{k}(S) \rightarrow \operatorname{PConf}_{k}(S) / G$. For $S=D^{2}$ (the open disk) and $G=S_{k}$, the space $\operatorname{Conf}_{k}\left(D^{2}\right):=\operatorname{PConf}_{k}\left(D^{2}\right) / S_{k}$ has fundamental group given by the classical braid group

$$
B_{k}:=\pi_{1}\left(\operatorname{Conf}_{k}\left(D^{2}\right)\right)
$$

The primary surface of interest in this paper is the Riemann sphere $S=S^{2}$. There are two subgroups $G$ as above that will be of interest. First is $G=S_{k}$. We write

$$
\operatorname{Conf}_{k}\left(S^{2}\right):=\operatorname{PConf}_{k}\left(S^{2}\right) / S_{k} ;
$$

this is the space of unordered $k$-tuples of distinct points on $S^{2}$. The spherical braid group is defined to be the fundamental group of this space:

$$
B_{k}\left(S^{2}\right):=\pi_{1}\left(\operatorname{Conf}_{k}\left(S^{2}\right)\right) .
$$

Secondly, suppose $k=n+m$, and consider the subgroup $G=S_{n} \times S_{m}$ of $S_{k}$. Write

$$
\operatorname{Conf}_{n, m}\left(S^{2}\right):=\operatorname{PConf}_{k}\left(S^{2}\right) /\left(S_{n} \times S_{m}\right) .
$$

This can be viewed as the space of $n$ "red" points and $m$ "blue" points which are otherwise indistinguishable. This leads to a useful piece of terminology.

Definition 2.1 (old points, new points) Relative to the preceding discussion, we refer to the set of cardinality $n$ as the set of old points, and the set of cardinality $m$ as the set of new points. The set of old points is written $\left\{x_{1}, \ldots, x_{n}\right\}$, and the set of new points is written $\left\{y_{1}, \ldots, y_{m}\right\}$.

We define

$$
B_{n, m}\left(S^{2}\right):=\pi_{1}\left(\operatorname{Conf}_{n, m}\left(S^{2}\right)\right)
$$

There is an evident forgetful map $P: \operatorname{Conf}_{n, m}\left(S^{2}\right) \rightarrow \operatorname{Conf}_{n}\left(S^{2}\right)$ giving rise to a surjective homomorphism

$$
p: B_{n, m}\left(S^{2}\right) \rightarrow B_{n}\left(S^{2}\right) .
$$

It is this $p$ that we seek to find (obstructions to) sections of. Recall that a section of a surjective group homomorphism $p: A \rightarrow B$ is a (necessarily injective) homomorphism $s: B \rightarrow A$ satisfying $p \circ s=\mathrm{id}$. 


\subsection{Spherical braid groups and the configuration spaces}

Before proving Theorem B, we first discuss an assertion mentioned in the introduction, where we claimed that a section $s: B_{n}\left(S^{2}\right) \rightarrow B_{n, m}\left(S^{2}\right)$ can be promoted to a section $S: \operatorname{Conf}_{n}\left(S^{2}\right) \rightarrow \operatorname{Conf}_{n, m}\left(S^{2}\right)$. This is a standard argument in obstruction theory; see [10, Proposition 4] for a written account.

Proposition 2.2 (Gonçalves-Guaschi) For $n \geq 3$, the fiber bundle Conf ${ }_{n, m}\left(S^{2}\right) \rightarrow$ $\operatorname{Conf}_{n}\left(S^{2}\right)$ admits a section if and only if there is a group-theoretic section $s: B_{n}\left(S^{2}\right) \rightarrow$ $B_{n, m}\left(S^{2}\right)$.

\subsection{A presentation of the spherical braid group}

It is classically known that $B_{n}\left(S^{2}\right)$ has a presentation obtained by adding a single relation to Artin's presentation of the classical braid group $B_{n}$; see [11, Theorem 32]. Let $R_{n}$ be the word in $\sigma_{1}, \ldots, \sigma_{n-1}$ given by

$$
R_{n}=\sigma_{1} \cdots \sigma_{n-1} \sigma_{n-1} \cdots \sigma_{1} .
$$

Then $B_{n}\left(S^{2}\right)$ has the following presentation:

$B_{n}\left(S^{2}\right)=$ $\left\langle\sigma_{1}, \ldots, \sigma_{n-1}\right|\left[\sigma_{i}, \sigma_{j}\right]=1$ for $|i-j|>1, \sigma_{i} \sigma_{i+1} \sigma_{i}=\sigma_{i+1} \sigma_{i} \sigma_{i+1}$ for $1 \leq i \leq n-2$,$$
\left.R_{n}=1\right\rangle \text {. }
$$

The element $R_{n}$ is depicted in Figure 1.

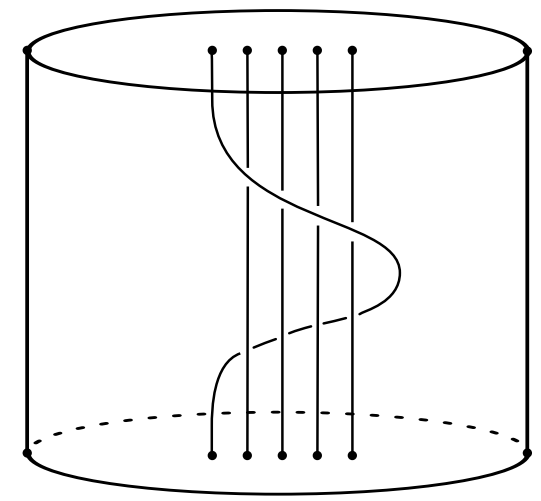

Figure 1: The braid $R_{n}=\sigma_{1} \cdots \sigma_{n-1}^{2} \cdots \sigma_{1} \subset D^{2} \times[0,1]$. 


\subsection{Torsion in the spherical braid group}

In [15], Murasugi determined the finite-order elements in $B_{n}\left(S^{2}\right)$. He showed that every finite-order element is conjugate to a power of one of the following three elements. Their properties are summarized in the table below:

\begin{tabular}{|c|ccc|}
\hline element & expression & order & permutation \\
\hline$\alpha_{0}$ & $\sigma_{1} \cdots \sigma_{n-1}$ & $2 n$ & $(1 \ldots n)$ \\
$\alpha_{1}$ & $\sigma_{1} \cdots \sigma_{n-2} \sigma_{n-1}^{2}$ & $2 n-2$ & $(1 \ldots n-1)$ \\
$\alpha_{2}$ & $\sigma_{1} \cdots \sigma_{n-3} \sigma_{n-2}^{2}$ & $2 n-4$ & $(1 \ldots n-2)$ \\
\hline
\end{tabular}

Remark 2.3 From the table, one can easily determine the permutation associated to any power of (a conjugate of) $\alpha_{i}$. Explicitly, $\alpha_{i}^{k}$ has permutation given by $(1 \ldots n-i)^{k}$, which decomposes into $\operatorname{gcd}(k, n-i)$ disjoint $j$-cycles, where

$$
j=\frac{n-i}{\operatorname{gcd}(k, n-i)} \text {. }
$$

\subsection{An alternative generating set}

Although the set $\left\{\sigma_{1}, \ldots, \sigma_{n-1}\right\}$ of "standard" generators for $B_{n}$ is the most widely known, it is not the most useful for our purposes. The starting point for this discussion is the following elementary lemma; see [11, Equation 24] or [9, Lemma 1(d)].

Lemma 2.4 For $1 \leq i \leq n-2$, there is an equality

$$
\alpha_{0}^{i} \sigma_{1} \alpha_{0}^{-i}=\sigma_{1+i}
$$

of elements of $B_{n}$. Similarly, for $1 \leq i \leq n-3$, there is an equality

$$
\alpha_{1}^{i} \sigma_{1} \alpha_{1}^{-i}=\sigma_{1+i}
$$

As an immediate corollary, we obtain our desired generating set, previously observed in $[9$, Lemma $1(d)]$.

Lemma 2.5 $B_{n}$, and hence its quotient $B_{n}\left(S^{2}\right)$, is generated by the set $\left\{\sigma_{1}, \alpha_{0}\right\}$.

We will also have occasion to study the subgroup generated by the elements $\alpha_{1}$ and $\sigma_{1}$. This group admits the following convenient description. 
Lemma 2.6 Let $B_{n-1,1}\left(S^{2}\right) \leqslant B_{n}\left(S^{2}\right)$ be the subgroup consisting of braids that fix the point $x_{n}$. Then $B_{n-1,1}\left(S^{2}\right)$ is generated by the set $\left\{\sigma_{1}, \alpha_{1}\right\}$.

Proof Given Lemma 2.4, it suffices to show that $\sigma_{1}, \ldots, \sigma_{n-2}$ generates $B_{n-1,1}\left(S^{2}\right)$. Let $P_{n}\left(S^{2}\right)$ be the kernel of the map $p: B_{n}\left(S^{2}\right) \rightarrow S_{n}$ recording the permutation of points. There is a short exact sequence

$$
1 \rightarrow P_{n}\left(S^{2}\right) \rightarrow B_{n-1,1}\left(S^{2}\right) \stackrel{\pi}{\longrightarrow} S_{n-1} \rightarrow 1 .
$$

As $\left\{\pi\left(\sigma_{1}\right), \ldots, \pi\left(\sigma_{n-2}\right)\right\}$ generates $S_{n-1}$, it suffices to show that the kernel $P_{n}\left(S^{2}\right)$ is contained in the subgroup $H=\left\langle\sigma_{1}, \ldots, \sigma_{n-2}\right\rangle$ of $B_{n-1,1}\left(S^{2}\right)$. Since $R_{n}=1 \in$ $B_{n}\left(S^{2}\right)$, it follows that $\sigma_{n-1}^{2} \in H$. Let $P_{n}$ be the kernel of the map $B_{n} \rightarrow S_{n}$ recording the permutation of points. We define the subgroup $G \leqslant B_{n}$ by

$$
G:=\left\langle\sigma_{1}, \ldots, \sigma_{n-2}, \sigma_{n-1}^{2}\right\rangle .
$$

Let $q: B_{n} \rightarrow B_{n}\left(S^{2}\right)$ be the natural projection from the classical braid group to the spherical braid group. By definition, $q(G)=H$. For $1 \leq i<j \leq n-1$, we define

$$
A_{i, j}=\sigma_{i}^{-1} \sigma_{i+1}^{-1} \cdots \sigma_{j-2}^{-1} \sigma_{j-1}^{2} \sigma_{j-2} \cdots \sigma_{i} .
$$

According to Artin [1, Theorem 17], the set $\left\{A_{i, j}\right\}$ generates $P_{n}$, and evidently $\left\{A_{i, j}\right\} \subset G$, so that $P_{n} \leqslant G$. Therefore we have that $q\left(P_{n}\right) \leqslant H$. Since we know that $q\left(P_{n}\right)=P_{n}\left(S^{2}\right)$, we get that $P_{n}\left(S^{2}\right) \leqslant H$.

\subsection{From braid groups to mapping class groups}

Let $S$ be a surface and let $\operatorname{Mod}_{n}(S)$ denote the mapping class group of $S$ relative to $n$ unordered marked points (equivalently the marked points can be viewed as punctures). We also define $\operatorname{Mod}_{n, m}(S)$ as the subgroup of $\operatorname{Mod}_{n+m}(S)$ consisting of mapping classes that preserve a partitioning of the marked points into two sets of cardinalities $n$ and $m$, respectively. We emphasize that mapping classes in $\operatorname{Mod}_{n, m}(S)$ are free to internally permute the points of the $n$-element and $m$-element sets, but are prohibited from exchanging points from one set for another. Also recall the standard convention, to be used throughout, that all isotopies are required to fix all marked points, and that isotopies of curves, subsurfaces, etc on $S$ must be induced from ambient isotopies of $S$ that preserve marked points.

The "point-pushing construction" (see eg [8, Chapter 9.1.4]) yields a homomorphism

$$
\mathcal{P}: B_{n}(S) \rightarrow \operatorname{Mod}_{n}(S) \text {. }
$$


For most surfaces, $\mathcal{P}$ is an isomorphism onto its image, but this is not the case for $S=S^{2}$. Rather, there is the short exact sequence

$$
1 \rightarrow \mathbb{Z} / 2 \mathbb{Z} \rightarrow B_{n}\left(S^{2}\right) \stackrel{\mathcal{P}}{\longrightarrow} \operatorname{Mod}_{n}\left(S^{2}\right) \rightarrow 1
$$

(again, see [8, Chapter 9.1.4]). The nontrivial element of the kernel is the central element $\omega$. It is characterized by the property that it is the unique element of order 2 in $B_{n}\left(S^{2}\right)$.

Lemma 2.7 [11, Proposition 33] There is a unique element $\omega \in B_{n}\left(S^{2}\right)$ of order 2 .

The classification of torsion in $B_{n}\left(S^{2}\right)$ given in (2) ports directly over to $\operatorname{Mod}_{n}(S)$. The only difference between torsion in the mapping class group is that the element $\alpha_{i}$ has order $n-i$ as opposed to $2 n-2 i$. As mapping classes, the torsion elements $\alpha_{i}$ have simple geometric representatives. Arrange the points $x_{1}, \ldots, x_{n-1-i}$ at equal intervals on the equator, and place any remaining points at the north and south poles. As a mapping class, $\alpha_{i}$ is then represented by a rotation of the sphere by an angle of $2 \pi /(n-i)$ through the plane of the equator.

It is a priori possible that a section $s: B_{n}\left(S^{2}\right) \rightarrow B_{n, m}\left(S^{2}\right)$ could fail to descend to a section $\bar{s}: \operatorname{Mod}_{n}\left(S^{2}\right) \rightarrow \operatorname{Mod}_{n, m}\left(S^{2}\right)$. If this were the case, we would not be able to prove Theorem A by moving to the setting of the mapping class group. However, we show here that this is not the case.

Lemma 2.8 Suppose that $s: B_{n}\left(S^{2}\right) \rightarrow B_{n, m}\left(S^{2}\right)$ is a section. Then there is a section $\bar{s}: \operatorname{Mod}_{n}\left(S^{2}\right) \rightarrow \operatorname{Mod}_{n, m}\left(S^{2}\right)$.

Proof The relationship between the four groups under study is summarized by the following diagram, where the rows are short exact sequences (here $\left\langle\omega_{n}\right\rangle$ denotes the subgroup generated by $\omega_{n}$ ):

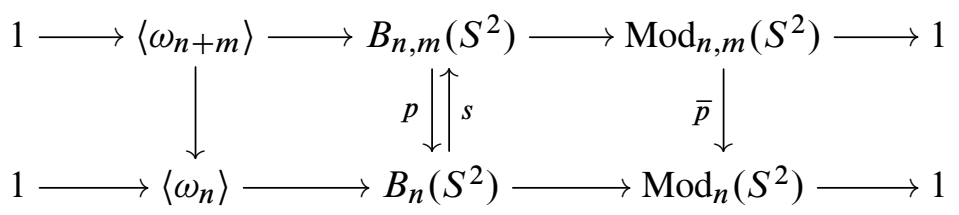

Given $f \in \operatorname{Mod}_{n}\left(S^{2}\right)$, let $\tilde{f} \in B_{n}\left(S^{2}\right)$ be an arbitrary lift. We claim that

$$
\bar{s}(f):=s(\tilde{f}) \quad \bmod \left\langle\omega_{n+m}\right\rangle
$$


gives a well-defined section homomorphism. To see this, recall from Lemma 2.7 that $\omega_{n} \in B_{n}\left(S^{2}\right)$ is the unique element of order 2 in $B_{n}\left(S^{2}\right)$, and that $\omega_{n+m}$ is similarly characterized as an element of $B_{n+m}\left(S^{2}\right)$. Since $s$ is a section, it follows that $s\left(\omega_{n}\right)=\omega_{n+m}$. Thus $\bar{s}(f)$ is well-defined as an element of

$$
B_{n, m}\left(S^{2}\right) /\left\langle\omega_{n+m}\right\rangle \cong \operatorname{Mod}_{n, m}\left(S^{2}\right) .
$$

It is straightforward to verify that $\bar{s}$ determines a homomorphism and that $\bar{p} \circ \bar{s}=\mathrm{id}$.

\section{Cabling braids on the sphere}

This section is dedicated to the proof of Theorem B. We begin with a discussion of the cabling construction. We then analyze the obstruction to cabling in the setting of the spherical braid group, leading to the proof of Theorem B.

Cabling crops up in many aspects of the theory of braids; see eg [16; 5]. To the authors' knowledge, the theory of cabling in the spherical braid group has not been treated in the literature, so we give here a self-contained account.

\subsection{Cabling}

Cabling has a simple intuitive description. Given a braid $\beta$, one imagines each strand as actually being composed of a cable of smaller strands. This should furnish a homomorphism $c_{k}: B_{n} \rightarrow B_{n k}$, where $k$ is the number of strands in each cable. Making this precise requires additional data: one must specify the internal braid structure that each cable possesses.

Definition 3.1 (cabling vector) Let $\phi \in B_{k}$ be fixed, and let $a_{1}, \ldots, a_{n-1}, c, t$ be arbitrary integers. The cabling vector is the tuple $v \in B_{k} \times \mathbb{Z}^{n+1}$ given by

$$
v:=\left(\phi ; a_{1}, \ldots, a_{n-1}, c, t\right) .
$$

Remark 3.2 As we are ultimately interested in constructing sections of $B_{n, m}\left(S^{2}\right) \rightarrow$ $B_{n}\left(S^{2}\right)$, we will need to understand when it is possible to "de-cable", that is, when there exists a homomorphism $p: B_{n k} \rightarrow B_{n}$ such that $p \circ c_{v}=\mathrm{id}$. It is geometrically clear that such a $p$ exists whenever the element $\phi$ is contained in the subgroup $B_{k-1,1}$ consisting of braids where one strand is required to return to its starting point. In this case, $c_{v}$ is valued in the subgroup $B_{n, n(k-1)}$. 

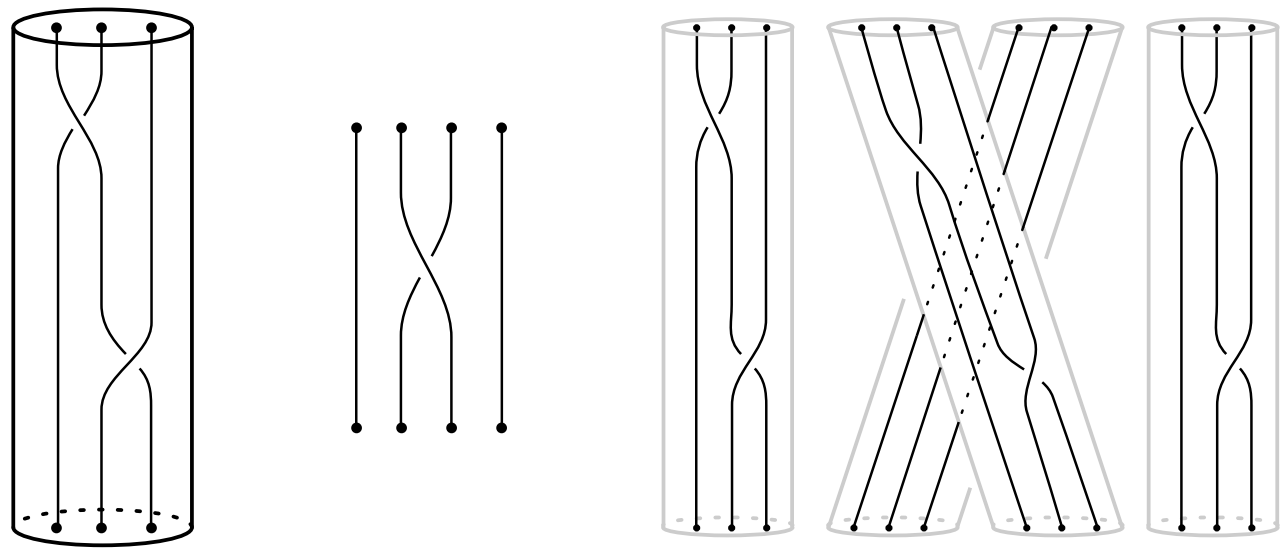

Figure 2: The cabling procedure. Left to right: the braid $\phi \in B_{3}$, the generator $\sigma_{2}$, and the cabled braid $c_{v}\left(\sigma_{2}\right)$. Here $a_{2}=1, c=1, t=1$.

Lemma 3.3 (cabling construction) Let $v=\left(\phi ; a_{1}, \ldots, a_{n-1}, c, t\right)$ be a cabling vector. Then there is a homomorphism

$$
c_{v}: B_{n} \rightarrow B_{n k} .
$$

Under $c_{v}$, each generator $\sigma_{i}$ is replaced by a cable: the $i^{\text {th }}$ strand is replaced with the braid $\phi^{a_{i}}$, the $(i+1)^{\text {st }}$ is replaced with $\phi^{t-a_{i}}$, and all remaining strands are replaced with the braid $\phi^{c}$.

Proof To prove the lemma, it suffices to show that the cabled generators $\left\{c_{v}\left(\sigma_{i}\right)\right\}$ satisfy all braid and commutation relations. These are both straightforward to check. For instance, consider the two braids $c_{v}\left(\sigma_{i} \sigma_{i+1} \sigma_{i}\right)$ and $c_{v}\left(\sigma_{i+1} \sigma_{i} \sigma_{i+1}\right)$. In both braids, the $i^{\text {th }}$ strand is replaced by the braid $\phi^{a_{i}+a_{i+1}+c}$, the $(i+1)^{\text {st }}$ is replaced by $\phi^{t+c}$, the $(i+2)^{\text {nd }}$ is replaced by $\phi^{2 t+c-a_{i}-a_{i+1}}$, and all other strands are replaced by $\phi^{3 c}$. The commutation relation $c_{v}\left(\sigma_{i} \sigma_{j}\right)=c_{v}\left(\sigma_{j} \sigma_{i}\right)$ for $|i-j|>1$ is similarly easy to verify.

\subsection{Cabling spherical braids}

The cabling construction described above makes implicit use of a consistent framing of a neighborhood of each strand. Such framings are trivial to construct when the ambient space is the disk, but the usual topological constraints obstruct such framings for spherical braids. On the group-theoretic level, this obstruction can be formulated in 
terms of the following diagram, whose rows are the short exact sequences describing the spherical braid groups as quotients of the braid groups of the disk:

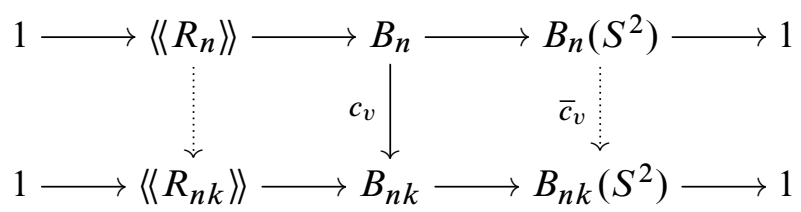

The diagram shows that the homomorphism $c_{v}: B_{n} \rightarrow B_{n k}$ will descend to a homomorphism $\bar{c}_{v}: B_{n}\left(S^{2}\right) \rightarrow B_{n k}\left(S^{2}\right)$ if and only if the braid $R_{n}=\sigma_{1} \cdots \sigma_{n-1}^{2} \cdots \sigma_{1}$ is sent to an element of $\left\langle\left\langle R_{n k}\right\rangle\right\rangle$ (the normal closure of the element $R_{n k}$ ). In geometric terms, this is equivalent to the requirement that $c_{v}\left(R_{n}\right)$ be isotopic to the identity as a spherical braid.

In order to understand the constraints this imposes on the cabling vector, we must understand the isotopy between the spherical braids $R_{n}$ and 1 once framings are taken into account. The content of Lemma 3.4 below is that the isotopy $R_{n} \sim 1$ introduces a double twist to the framing. To study this, we introduce the braid $R_{n}(k)$ shown and defined in Figure 3.

Lemma 3.4 For any $n \geq 3$ and $k \geq 2$, the braid $R_{n}(k)$ of Figure 3 is trivial as an element of $B_{n k}\left(S^{2}\right)$.

Proof This can be directly verified by applying the isotopy shown in Figure 4.

The braid $R_{n}(k)$ is a $k$-stranded cabling of $R_{n}$. In order for a given cabling homomorphism $c_{v}: B_{n} \rightarrow B_{n k}$ to descend to $\bar{c}_{v}: B_{n}\left(S^{2}\right) \rightarrow B_{n k}\left(S^{2}\right)$, it suffices to produce a cabling vector $v=\left(\phi, a_{1}, \ldots, a_{n-1}, c, t\right)$ for which $c_{v}\left(R_{n}\right)=R_{n}(k)$. Lemma 3.5 produces a class of suitable such $v$.

Lemma 3.5 Let $k=k^{\prime}(n-1)(n-2)+1$, and let $\phi \in B_{k-1,1}$ be the element $\phi=\left(\sigma_{1} \cdots \sigma_{k-2} \sigma_{k-1}^{2}\right)^{k^{\prime}}$. For $a_{1}, \ldots, a_{n-1}$ arbitrary, $c=-1$, and $t=2 n-4$, the cabling vector $v=\left(\phi, a_{1}, \ldots, a_{n-1}, c, t\right)$ satisfies

$$
c_{v}\left(R_{n}\right)=R_{n}(k)
$$

Proof We must analyze the internal braiding on each strand of the cabled braid

$$
c_{v}\left(R_{n}\right)=c_{v}\left(\sigma_{1} \cdots \sigma_{n-1}^{2} \cdots \sigma_{1}\right)
$$




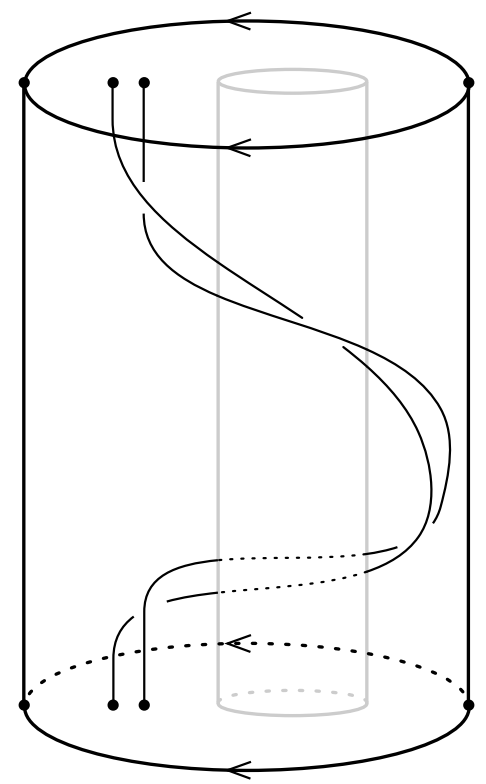

Figure 3: The braid $R_{n}(k) \subset S^{2} \times[0,1]$, depicted for $k=2$. The remaining $(n-1) k$ strands are contained inside the gray cylinder and have constant $S^{2}$-coordinate. For general $k$, one can imagine the two depicted strands as determining the left and right edges of a flat strip on which the $k$ strands are arranged.

In order to determine $c_{v}\left(R_{n}\right)$, we track one strand at a time, applying the cabling construction (Lemma 3.3) one letter at a time.

We begin with the first strand. Before applying the $i^{\text {th }}$ letter of the subword $\sigma_{1} \cdots \sigma_{n-1}$, the first strand is in position $i$, and so is cabled with $\phi^{a_{i}}$. Before applying the $i^{\text {th }}$ letter of the other subword $\sigma_{n-1} \cdots \sigma_{1}$, the first strand is in position $n+1-i$, and so is cabled with $\phi^{t-a_{i}}$.

Altogether then, the first strand is cabled with $\phi^{(n-1) t}=\phi^{2(n-1)(n-2)}$. Geometrically, $\phi$ is represented as a rotation by an angle $2 \pi /(n-1)(n-2)$, with one strand fixed at the center of the disk and the remaining strands arranged at equally spaced points along a circle. Thus, $\phi^{2(n-1)(n-2)}$ is the braid given by two full twists of the strands about the central axis, which is exactly the cabling of the first strand in the braid $R_{n}(k)$.

The cabling on the remaining strands can be determined in a similar fashion. Fix $j \geq 2$. The subword $\sigma_{1} \cdots \sigma_{j-2}$ leaves the $j^{\text {th }}$ strand in position $j$, and so gives a total cabling of $\phi^{2-j}$. Then $\sigma_{j-1}$ moves the $j^{\text {th }}$ strand to position $j-1$ and appends $\phi^{t-a_{j-1}}$ to 

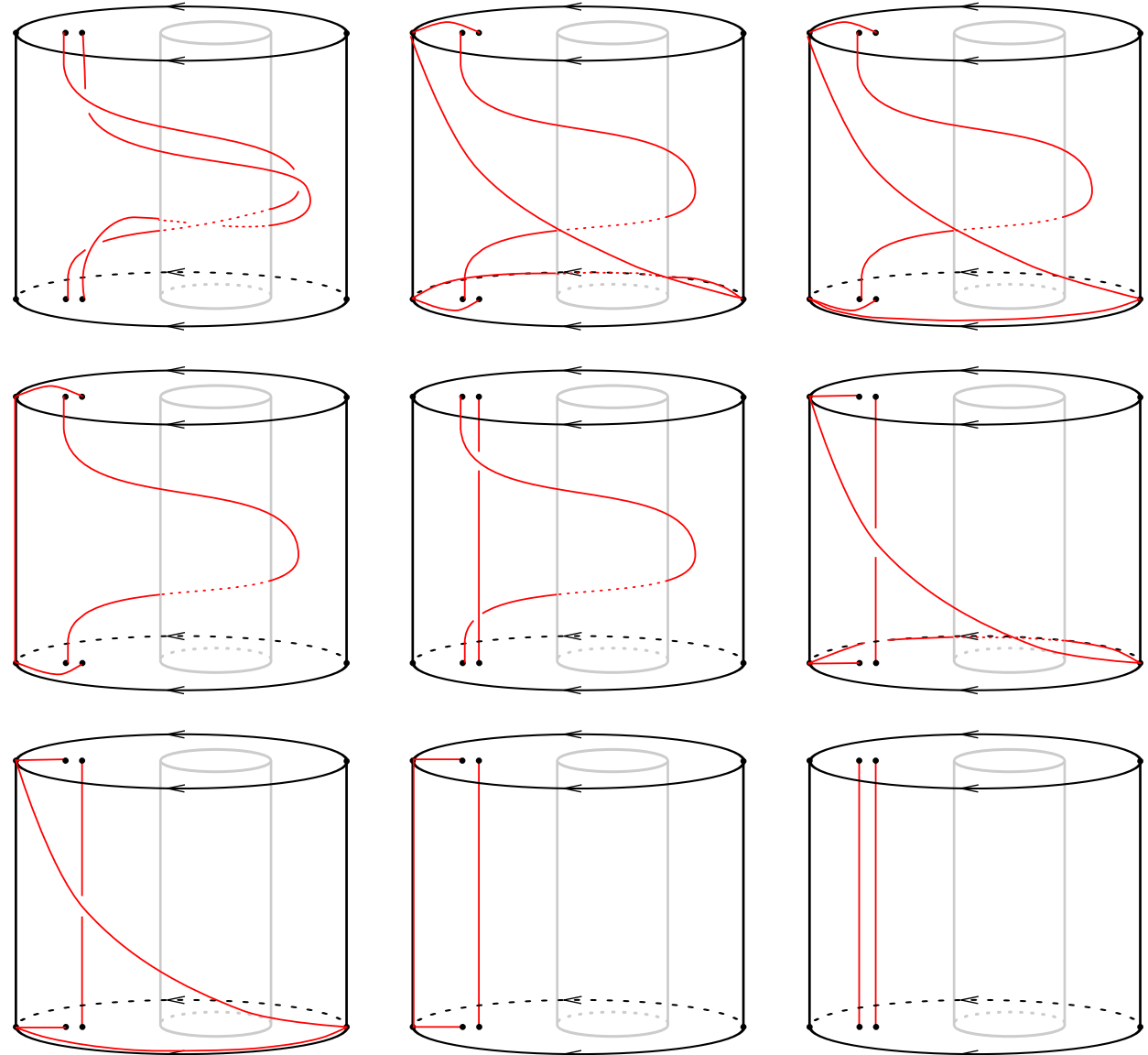

Figure 4: The isotopy of Lemma 3.4. The sequence should be read lexicographically. Between steps 2 and 3, a portion of the braid is pulled from the back to the front using the edge identification; the same move occurs between steps 6 and 7.

the cabling. The subword $\sigma_{j} \cdots \sigma_{n-1}^{2} \cdots \sigma_{j}$ leaves the $j^{\text {th }}$ strand in position $j-1$, appending $\phi^{2 j-2 n}$. Then $\sigma_{j-1}$ moves the $j^{\text {th }}$ strand to position $j$, appending $\phi^{a_{j-1}}$ to the cabling. Finally the remaining subword $\sigma_{j-2} \cdots \sigma_{1}$ appends an additional $\phi^{2-j}$. The total cabling induced by this procedure is $\phi^{t+4-2 n}=\mathrm{id}$, since $t=2 n-4$. Thus, $c_{v}\left(R_{n}\right)=R_{n}(k)$, as claimed.

Theorem B now follows from the preceding analysis.

Proof of Theorem B Suppose $m$ is divisible by $n(n-1)(n-2)$, and set $k=m / n+1$. Let $v$ be a cabling vector satisfying the hypotheses of Lemma 3.5. Applying Lemma 3.5 
and appealing to (the analysis following) diagram (3), it follows that $c_{v}$ descends to a homomorphism

$$
\bar{c}_{v}: B_{n}\left(S^{2}\right) \rightarrow B_{n k}\left(S^{2}\right) .
$$

Note that $\phi=\left(\sigma_{1} \cdots \sigma_{k-2} \sigma_{k-1}^{2}\right)^{k^{\prime}}$ is an element of $B_{k-1,1}$. By Remark 3.2, $\bar{c}_{v}$ is valued in the subgroup $B_{n, n(k-1)}\left(S^{2}\right)=B_{n, m}\left(S^{2}\right)$ and so provides a section of $p: B_{n, m}\left(S^{2}\right) \rightarrow B_{n}\left(S^{2}\right)$ as desired. By Proposition 2.2, $\bar{c}_{v}$ can be promoted to a section $C_{v}$ : $\operatorname{Conf}_{n}\left(S^{2}\right) \rightarrow \operatorname{Conf}_{n, m}\left(S^{2}\right)$.

Remark 3.6 (cabling via rational maps) There is also an explicit construction of a section $S: \operatorname{Conf}_{n}\left(S^{2}\right) \rightarrow \operatorname{Conf}_{n, m}\left(S^{2}\right)$ that induces the cabling map $s: B_{n}\left(S^{2}\right) \rightarrow$ $B_{n, m}\left(S^{2}\right)$. To construct this, given an ordered triple $\left(z_{1}, z_{2}, z_{3}\right)$ of distinct points in $\mathbb{C P}^{1}$, we let $M_{z_{1}, z_{2}, z_{3}}$ be the unique Möbius transformation taking $\left(z_{1}, z_{2}, z_{3}\right) \rightarrow$ $(0,1, \infty)$. This determines a map

$$
M: \operatorname{Conf}_{n-3,1^{3}}\left(\mathbb{C P}^{1}\right) \times \mathbb{C P}^{1} \rightarrow \mathbb{C P}^{1},
$$

where $\operatorname{Conf}_{n-3,1^{3}}\left(\mathbb{C P}^{1}\right)$ is the cover of $\operatorname{Conf}_{n}\left(\mathbb{C P}^{1}\right)$ consisting of configurations with three ordered distinguished points. At $\left(\left\{z_{4}, \ldots, z_{n}\right\}, z_{1}, z_{2}, z_{3}\right) \times \mathbb{C P}^{1}$, the map $M$ is given by $M_{z_{1}, z_{2}, z_{3}}$.

For a fixed $\left(\left\{z_{4}, \ldots, z_{n}\right\}, z_{1}, z_{2}, z_{3}\right) \in \operatorname{Conf}_{n-3,1^{3}}\left(\mathbb{C P}^{1}\right)$, the product

$$
R_{z_{1}}:=\prod_{i \neq j \in\{2, \ldots, n\}} M_{z_{1}, z_{i}, z_{j}}
$$

determines a rational map of degree $(n-1)(n-2)$ with a zero at $z_{1}$ of order $(n-1)(n-2)$. We define $R_{z_{i}}$ for $1 \leq i \leq n$ similarly. By construction, each $R_{z_{i}}$ is defined at the level of the configuration space $\operatorname{Conf}_{n-1,1}\left(S^{2}\right)$.

To construct the cable near the point $z_{i}$, we take the inverse image $R_{z_{i}}^{-1}(\varepsilon)$ for some small $\varepsilon$ a regular value of $R_{z_{i}}$. Taking the union of these collections of points for $R_{z_{1}}, \ldots, R_{z_{n}}$ gives the cabling section. There is a certain amount of care needed to define $\varepsilon$ suitably; the details are below.

There is a continuous function $r_{i}$ : $\operatorname{Conf}_{n-1,1}\left(S^{2}\right) \rightarrow \mathbb{R}^{+}$such that under $R_{z_{i}}$, points in $\left\{0<|z|<r_{i}\right\}$ have $(n-1)(n-2)$ distinct preimages. Relative to the spherical metric $d$ on $S^{2}$, we define another function $\rho^{\prime}: \operatorname{Conf}_{n}\left(S^{2}\right) \rightarrow \mathbb{R}^{+}$such that

$$
\rho^{\prime}\left(\left\{z_{1}, \ldots, z_{n}\right\}\right):=\frac{1}{3} \min _{i \neq j} d\left(z_{i}, z_{j}\right) .
$$


Using $\rho^{\prime}$, we define another continuous function $\rho_{i}^{\prime}: \operatorname{Conf}_{n-1,1} \rightarrow \mathbb{R}^{+}$such that $R_{z_{i}}^{-1}\left(B\left(0, \rho_{i}\right)\right) \subset B\left(z_{i}, \rho^{\prime}\right)$. By taking the common minimum, we obtain $\rho_{i}\left(\left\{z_{1}, \ldots, \hat{z}_{i}, \ldots, z_{n}\right\}, z_{i}\right)$

$$
:=\min _{i}\left\{r_{i}\left(\left\{z_{1}, \ldots, \widehat{z}_{i}, \ldots, z_{n}\right\}, z_{i}\right), \rho_{i}^{\prime}\left(\left\{z_{1}, \ldots, \widehat{z}_{i}, \ldots, z_{n}\right\}, z_{i}\right)\right\} .
$$

Now, we define the section function

$$
S\left(\left\{z_{1}, \ldots, z_{n}\right\}\right)=\bigcup_{i=1}^{n} R_{z_{i}}^{-1}\left(\rho_{i}\left(\left\{z_{1}, \ldots, \widehat{z}_{i}, \ldots, z_{n}\right\}, z_{i}\right)\right) .
$$

By definition, $S_{i}:=R_{z_{i}}^{-1}\left(\rho_{i}\left(\left\{z_{1}, \ldots, \widehat{z}_{i}, \ldots, z_{n}\right\}, z_{i}\right)\right)$ contains $(n-1)(n-2)$ points and is contained in $B\left(z_{i}, \rho^{\prime}\right)$, which shows that $S_{1} \cup \cdots \cup S_{n}$ consists of exactly $n(n-1)(n-2)$ points. By taking $k$ different $\rho_{i}$ functions $\left(\right.$ eg $\left.\rho_{i}, \frac{1}{2} \rho_{i}, \ldots, \frac{1}{k} \rho_{i}\right)$, one can construct continuous sections $s: \operatorname{Conf}_{n}\left(\mathbb{C P}^{1}\right) \rightarrow \operatorname{Conf}_{n, m}\left(\mathbb{C P}^{1}\right)$ for any $m$ of the form $m=k n(n-1)(n-2)$.

\section{Three and four points}

In this section, we give some algebrogeometric constructions of sections of the bundles $\operatorname{Conf}_{3, m}\left(\mathbb{C P}^{1}\right) \rightarrow \mathrm{Conf}_{3}\left(\mathbb{C P}^{1}\right)$ and $\mathrm{Conf}_{4, m}\left(\mathbb{C P}^{1}\right) \rightarrow \mathrm{Conf}_{4}\left(\mathbb{C P}^{1}\right)$. These results are summarized in Theorem $\mathrm{C}$.

\subsection{Three points}

The space $\operatorname{Conf}_{3}\left(S^{2}\right)$ is of course very special. Under the identification $S^{2}=\mathbb{C P}^{1}$, the group $\operatorname{Aut}\left(\mathbb{C P}^{1}\right)=\mathrm{PGL}_{2}(\mathbb{C})$ acts triply transitively on $\mathbb{C P}^{1}$, ie transitively on $\operatorname{Conf}_{3}\left(\mathbb{C P}^{1}\right)$. Thus one method for constructing sections is to first apply an automorphism to normalize the configuration to the set $\{0,1, \infty\}$, and then find all possible configurations of points distinct from $\{0,1, \infty\}$ and invariant under the stabilizer of $\{0,1, \infty\}$ in $\operatorname{Aut}\left(\mathbb{C P}^{1}\right)$. We will flesh out this approach by means of the cross-ratio. Recall from Remark 3.6 the Möbius transformation $M_{z_{1}, z_{2}, z_{3}}$ characterized by sending the triple $\left(z_{1}, z_{2}, z_{3}\right)$ to $(0,1, \infty)$.

The cross-ratio Let $z_{1}, z_{2}, z_{3}, z_{4} \in \mathbb{C P}^{1}$ be four ordered points. The cross-ratio is the expression

$$
\left[z_{1}, z_{2} ; z_{3}, z_{4}\right]:=M_{z_{1}, z_{2}, z_{3}}\left(z_{4}\right)=\frac{\left(z_{2}-z_{3}\right)\left(z_{4}-z_{1}\right)}{\left(z_{2}-z_{1}\right)\left(z_{4}-z_{3}\right)} .
$$


To see how the cross-ratio can be exploited to construct sections of $\operatorname{Conf}_{3, n}\left(\mathbb{C P}^{1}\right) \rightarrow$ $\operatorname{Conf}_{3}\left(\mathbb{C P}^{1}\right)$, it is necessary to understand how the value of $\left[z_{1}, z_{2} ; z_{3}, z_{4}\right]$ changes under a permutation $\sigma \in S_{4}$.

Lemma 4.1 Let $z_{1}, z_{2}, z_{3}, z_{4} \in \mathbb{C P}^{1}$ be given, and suppose that $\left[z_{1}, z_{2} ; z_{3}, z_{4}\right]=\lambda$. Let $\sigma \in S_{4}$ be an arbitrary permutation. Then

$$
\left[z_{\sigma(1)}, z_{\sigma(2)} ; z_{\sigma(3)}, z_{\sigma(4)}\right] \in\left\{\lambda, \frac{1}{\lambda}, 1-\lambda, \frac{1}{1-\lambda}, \frac{\lambda-1}{\lambda}, \frac{\lambda}{\lambda-1}\right\} .
$$

Thus the cross-ratio determines a generically 6 -valued function $\left[\left\{z_{1}, z_{2}, z_{3}, z_{4}\right\}\right]$ of unordered 4-tuples.

Remark 4.2 It is easy to see that the stabilizer of $\{0,1, \infty\}$ in $\mathrm{PGL}_{2}(\mathbb{C})$ is the dihedral group $D_{3}$. The six values $\left\{\lambda, \frac{1}{\lambda}, 1-\lambda, \frac{1}{1-\lambda}, \frac{\lambda-1}{\lambda}, \frac{\lambda}{\lambda-1}\right\}$ in fact comprise the orbit of $\lambda$ under $D_{3}$.

Lemma 4.1 also allows us to view the cross-ratio as a multivalued function on $\operatorname{Conf}_{3}\left(\mathbb{C P}^{1}\right)$. Given $\left\{z_{1}, z_{2}, z_{3}\right\} \in \operatorname{Conf}_{3}\left(\mathbb{C P}^{1}\right)$ and $\lambda \in \mathbb{C P}^{1}$, define

$$
\times\left(z_{1}, z_{2}, z_{3}, \lambda\right)=\left\{z_{4} \in \mathbb{C P}^{1} \mid \lambda \in\left[\left\{z_{1}, z_{2}, z_{3}, z_{4}\right\}\right]\right\} .
$$

For generic values of $\lambda$, the function $\times$ is 6 -valued. However, $x$ is 3 -valued for $\lambda \in\left\{-1, \frac{1}{2}, 2\right\}$, and is 2 -valued for $\lambda=\zeta^{ \pm 1}$ either of the primitive sixth roots of unity. Moreover,

$$
\times\left(z_{1}, z_{2}, z_{3}, \lambda\right) \cap \times\left(z_{1}, z_{2}, z_{3}, \lambda^{\prime}\right)=\varnothing
$$

whenever $\lambda$ and $\lambda^{\prime}$ lie in different orbits of $D_{3}$, and

$$
\times\left(z_{1}, z_{2}, z_{3}, \lambda\right) \cap\left\{z_{1}, z_{2}, z_{3}\right\}=\varnothing
$$

as long as $\lambda \neq 0,1, \infty$.

Proposition 4.3 For any $m \geq 0$ satisfying $m \equiv 0,2 \bmod 3$, there exists an algebraic section $\sigma$ of the bundle $\operatorname{Conf}_{3, m}\left(\mathbb{C P}^{1}\right) \rightarrow \operatorname{Conf}_{3}\left(\mathbb{C P}^{1}\right)$. Moreover, $\sigma$ is conformally invariant, ie equivariant with respect to the action of $\mathrm{PGL}_{2}(\mathbb{C})$.

Proof There is a unique expression for $m$ of the form

$$
m=2 a+3 b+6 c
$$


with $a, b \in\{0,1\}$. Set $k=a+b+c$. Choose a set $\left\{\lambda_{1}, \ldots, \lambda_{k}\right\} \subset \mathbb{C P}^{1} \backslash\{0,1, \infty\}$; these points should lie in distinct orbits under the action of the stabilizer of $\{0,1, \infty\}$. If $a=1$ then set $\lambda_{1}=\zeta$; likewise, if $b=1$ then set $\lambda_{2}=-1$. Then the assignment

$$
\sigma\left(\left\{z_{1}, z_{2}, z_{3}\right\}\right)=\bigcup_{i=1}^{k} \times\left(z_{1}, z_{2}, z_{3}, \lambda_{i}\right)
$$

has the required properties.

\subsection{Four points}

We now turn to the problem of constructing sections of the bundle $\operatorname{Conf}_{4, m}\left(\mathbb{C P}^{1}\right) \rightarrow$ $\operatorname{Conf}_{4}\left(\mathbb{C P}^{1}\right)$. We are grateful to Ian Frankel for the suggestion to look at torsion points on elliptic curves. The basic fact underlying the constructions in this section is the following well-known result; see [12, Example 4.8.2].

Lemma 4.4 Let $S=\left\{z_{1}, z_{2}, z_{3}, z_{4}\right\} \subset \mathbb{C P}^{1}$ be an arbitrary 4-tuple of distinct points. Then there exists a unique smooth algebraic curve $E_{S}$ of genus 1 such that under the elliptic involution $\iota: E_{S} \rightarrow \mathbb{C P}^{1}$, the branch locus in $\mathbb{C P}^{1}$ is the set $S$. If an arbitrary basepoint $* \in \iota^{-1}(S)$ is chosen, then the preimage $\iota^{-1}(S)$ is the set of 2-torsion points of the elliptic curve $\left(E_{S}, *\right)$. This association is continuous (even holomorphic) on the corresponding moduli spaces.

Lemma 4.4 leads to the construction of sections of $\operatorname{Conf}_{4, m}\left(\mathbb{C P}^{1}\right) \rightarrow \operatorname{Conf}_{4}\left(\mathbb{C P}^{1}\right)$ for many values of $m$. To formulate the result, let $P(k)$ denote the number of primitive elements of the group $(\mathbb{Z} / k \mathbb{Z})^{2}$. An explicit formula for $P(k)$ can be obtained from the observation that $P\left(p^{k}\right)=p^{2 k}-p^{2 k-2}$ for any prime $p$, in combination with the fact that $P$ is evidently a multiplicative function.

Proposition 4.5 Let $m$ be a positive integer of the form $m=2 k^{2}-2$ or $m=\frac{1}{2} P(4 k)$ or $m=2\left(p^{2}+q^{2}\right)-4$ for coprime integers $p$ and $q$. Then there exists an algebraic section $\sigma$ of the bundle $\operatorname{Conf}_{4, m}\left(\mathbb{C P}^{1}\right) \rightarrow \operatorname{Conf}_{4}\left(\mathbb{C P}^{1}\right)$. Moreover, $\sigma$ is conformally invariant.

Proof First consider the case $m=2 k^{2}-2$. Let $S=\left\{z_{1}, z_{2}, z_{3}, z_{4}\right\} \in \operatorname{Conf}_{4}\left(\mathbb{C P}^{1}\right)$ be given, and let $\left(E_{S}, *\right)$ be the elliptic curve of Lemma 4.4, with $* \in \iota^{-1}(S)$ chosen arbitrarily. The $2 k$-torsion subgroup of $\left(E_{S}, *\right)$ has cardinality $4 k^{2}$. Since $2 k$ is even, it follows that this set does not depend on which of the four points $\iota^{-1}\left(z_{i}\right)$ is 
chosen as the identity element. Among these points, exactly four are the 2-torsion points $\iota^{-1}(S)$. The elliptic involution $x \mapsto-x$ restricts to a free involution on the remaining $4 k^{2}-4$ points. Under $\iota$, these points descend to a set of $2 k^{2}-2$ distinct points on $S^{2}$ that are necessarily disjoint from $S$. The continuity and conformality of this construction follow from Lemma 4.4.

The construction for $m=\frac{1}{2} P(4 k)$ proceeds along similar lines. The set of primitive $4 k$-torsion points is well-defined independently of the choice of origin among the points $\iota^{-1}(S)$, and has cardinality $P(4 k)$ by definition. As before, this descends under the elliptic involution to a set of cardinality $\frac{1}{2} P(4 k)$ in $S^{2}$.

The construction for $m=2\left(p^{2}+q^{2}\right)-4$ is yet another variant. If $p$ and $q$ are coprime, then the $2 p$-torsion subgroup intersects the $2 q$-torsion subgroup in the 2-torsion subgroup. On the elliptic curve, this determines a set of $4\left(p^{2}+q^{2}\right)-8$ points distinct from the 2-torsion points, leading to the section of size $2\left(p^{2}+q^{2}\right)-4$ on $\mathbb{C P}^{1}$.

The first few such values of $m$ are given by $m=6,16,22,24$, corresponding respectively to the 4-torsion, 6-torsion, 4- and 6-torsion, and primitive 8-torsion. It appears to be a fairly intricate problem in elementary number theory to determine if every $m \equiv 0,6,16,22 \bmod 24$ can be obtained from Proposition 4.5 or some further elaboration thereof. However, the stated results are sufficient to prove Theorem C.

Proof of Theorem $\mathbf{C}$ The assertions concerning the case $n=3$ are subsumed by Proposition 4.3. The assertions concerning $n=4$ follow readily from Proposition 4.5 and Theorem B. If $m \geq 0$ is congruent to one of the four allowable values $0,6,16,22$ $\bmod 24$, then one can produce a section $\operatorname{Conf}_{4}\left(S^{2}\right) \rightarrow \operatorname{Conf}_{4, m}\left(S^{2}\right)$ by combining the construction of Proposition 4.5 (for 1-torsion, 4-torsion, 6-torsion, and 4- and 6-torsion, respectively, for $0,6,16,22 \bmod 24)$ with the cabling construction of Theorem B.

\section{Canonical reduction systems}

The goal of this section is to outline the portion of the theory of canonical reduction systems needed for the proof of Theorem A. For general references on the NielsenThurston classification and on canonical reduction systems, see [8, Chapter 13; 2].

We first recall the Nielsen-Thurston classification of elements of $\operatorname{Mod}(S)$, where $S$ is an arbitrary surface of finite type. For this discussion, and for the remainder 
of the paper, we invoke the usual conventions concerning isotopy: by "curve", we really mean "isotopy class of curves", by "disjoint", we really mean "existence of disjoint isotopy class representatives", by "subsurface" we really mean "isotopy class of subsurfaces", etc. Recall that isotopies are required to pointwise fix all marked points on $S$, so that, for example, it is sensible to talk about a marked point being contained in an isotopy class of subsurface.

With these stipulations in place, the Nielsen-Thurston classification asserts that each $f \in \operatorname{Mod}(S)$ is exactly one of the following types: periodic, reducible, or pseudoAnosov. A mapping class $f$ is periodic if $f^{n}=\mathrm{id}$ for some $n \geq 1$, and is reducible if there is some essential multicurve $\gamma \subset S$ fixed (as a set, not necessarily componentwise) by $f$. Otherwise, $f$ is said to be pseudo-Anosov.

Definition 5.1 (canonical reduction system) Let $f \in \operatorname{Mod}(S)$ be given. A reduction system for $f$ is any essential multicurve $\gamma=\left\{c_{1}, \ldots, c_{n}\right\}$ fixed setwise by $f$. A reduction system is maximal if it is maximal with respect to inclusion of reduction systems for $f$. The canonical reduction system for $f$, written $\operatorname{CRS}(f)$, is defined to be the intersection of all maximal reduction systems for $f$.

Canonical reduction systems provide a sort of Jordan form for mapping classes. The role of Jordan blocks is played by the components of the cut-open surface

$$
S_{\mathrm{CRS}(f)}:=S \backslash \mathrm{CRS}(f) .
$$

The lemma below follows from [2, Theorem C]; see also [8, Corollary 13.3].

Lemma 5.2 Let $f \in \operatorname{Mod}(S)$ be given, and suppose that $f$ preserves some component $S_{i}$ of $S_{\mathrm{CRS}(f)}$ and so induces an element $f_{i} \in \operatorname{Mod}\left(S_{i}\right)$. Then $f_{i}$ is either periodic or else pseudo-Anosov.

Remark 5.3 In this paper, we are exclusively interested in the case where $S$ is a punctured sphere. Then each component $S_{i}$ is also a punctured sphere, and so the classification of torsion elements of $\operatorname{Mod}\left(S_{i}\right)$ given in the table (2) is applicable. In particular, we see that if $f_{i} \in \operatorname{Mod}\left(S_{i}\right)$ is periodic and fixes at least three punctures, then $f_{i}$ is trivial, and any remaining punctures in $S_{i}$ must also be fixed. We note that by our definitions, a boundary component of $S_{i}$ (when viewed as a subsurface of $S$ ) is treated as a puncture when $S_{i}$ is viewed as an abstract punctured sphere. 
Canonical reduction systems behave as expected under conjugation. We record the following lemma for later use; its proof is trivial.

Lemma 5.4 Let $f, g \in \operatorname{Mod}(S)$ be given. Then

$$
\operatorname{CRS}\left(f g f^{-1}\right)=f(\operatorname{CRS}(g)) \text {. }
$$

In particular, if $f$ and $g$ commute, then $f(\operatorname{CRS}(g))=\operatorname{CRS}(g)$.

If mapping classes $f, g$ commute, then $\operatorname{CRS}(f)$ and $\operatorname{CRS}(g)$ satisfy an especially nice relationship; see [3, Proposition 2.6].

Lemma 5.5 Suppose that $f, g \in \operatorname{Mod}(S)$ commute. Then each component of CRS $(g)$ is either also a component of $\operatorname{CRS}(f)$, or else is disjoint from each component of $\operatorname{CRS}(f)$.

We conclude this section with a useful lemma giving a criterion for the equality of two subsurfaces of a punctured sphere.

Lemma 5.6 Let $S$ and $S^{\prime}$ be two connected subsurfaces of a punctured sphere $\Sigma$. Suppose that the boundaries $\partial S$ and $\partial S^{\prime}$ have the same number of components, and that each component of $\partial S$ is either disjoint from each component of $\partial S^{\prime}$, or else is also a component of $\partial S^{\prime}$. Suppose further that no component of $\partial S^{\prime}$ is contained in the interior of $S$. If $S$ and $S^{\prime}$ contain the same number of punctures and there is a puncture $x$ contained in both $S$ and $S^{\prime}$, then in fact $S$ and $S^{\prime}$ determine the same isotopy class of subsurface.

Proof The Euler characteristic of either surface is determined by the number of boundary components and the number of punctures contained in the interior. As each surface is a punctured sphere, it follows that moreover, the homeomorphism type is determined by this data, and hence the assumptions imply that $S$ and $S^{\prime}$ are abstractly homeomorphic. Since no component of $\partial S^{\prime}$ is contained in the interior of $S$, and since $S$ and $S^{\prime}$ contain some common puncture, it follows that there is a containment of subsurfaces $S \subset S^{\prime}$. Since $S$ and $S^{\prime}$ are assumed to contain the same number of punctures, these must each be contained in $S$. Therefore $S^{\prime}-S$ is a union of annuli, each of which contains no punctures. This means that $S$ and $S^{\prime}$ are isotopic subsurfaces. 


\section{Proof of Theorem A: preliminaries}

This is the first of five sections dedicated to the proof of Theorem A. The plan is as follows. In Section 6, we establish some preliminary ideas. This allows us to give a high-level overview of the proof in Section 7 and to divide the ensuing argument up into two cases, A and B. In Section 8 we prove a pair of crucial lemmas. The arguments for cases A and B are carried out in Sections 9 and 10, respectively.

Throughout the proof, fix $n \geq 6$. We remind the reader of the terminology of "old points" $\left\{x_{1}, \ldots, x_{n}\right\}$ and "new points" $\left\{y_{1}, \ldots, y_{m}\right\}$ of Definition 2.1. For the sake of contradiction, we assume that $m$ is the least integer not divisible by $n(n-1)(n-2)$ for which a section $s: B_{n}\left(S^{2}\right) \rightarrow B_{n, m}\left(S^{2}\right)$ exists. By Lemma 2.8, a section $s: B_{n}\left(S^{2}\right) \rightarrow$ $B_{n, m}\left(S^{2}\right)$ induces a section $s: \operatorname{Mod}_{n}\left(S^{2}\right) \rightarrow \operatorname{Mod}_{n, m}\left(S^{2}\right)$. We remind the reader that the group $\operatorname{Mod}_{n, m}\left(S^{2}\right)$ is allowed to permute points internally within the $n$-element and $m$-element sets, but cannot exchange a point in the $n$-element set for one in the $m$-element set. That is, $\operatorname{Mod}_{n, m}\left(S^{2}\right)$ preserves the sets of old and new points setwise.

For the remainder of the proof, we will work in the setting of the mapping class group. We define

$$
\Gamma:=s\left(\operatorname{Mod}_{n}\left(S^{2}\right)\right) \leqslant \operatorname{Mod}_{n, m}\left(S^{2}\right) .
$$

Before we can give the overview of the proof in the next section, there are three preliminary results that need to be established. In Section 6.1, we show that $\Gamma$ acts transitively on the set of new points (Lemma 6.2). In Section 6.2, we show that some torsion element fixes a new point (Lemma 6.3). Finally in Section 6.3, we study the canonical reduction system $\operatorname{CRS}\left(s\left(\sigma_{1}\right)\right)$ and attach to this a tree in a canonical way (Lemma 6.5).

Remark 6.1 (Where does the argument fail for $n=5$ ?) For the sake of future work, we document here exactly which portions of the argument are not valid for $n=5$. There are three such places: Case A.2.b in Section 9, and Lemmas 10.1 and 10.4 in Section 10.

\subsection{Transitivity on new points}

A first observation to be made is that our hypotheses on $m$ imply that the action of $\Gamma$ on the set of new points is transitive. 
Lemma 6.2 Let $m$ be the minimal integer not divisible by $n(n-1)(n-2)$ for which a section $s: \operatorname{Mod}_{n}\left(S^{2}\right) \rightarrow \operatorname{Mod}_{n, m}\left(S^{2}\right)$ exists. Then $\Gamma$ acts transitively on the set of new points.

Proof If $\Gamma$ does not act transitively on the set of new points, then there exists some nontrivial $\Gamma$-invariant partition of $\left\{y_{1}, \ldots, y_{m}\right\}$. Let $m^{\prime}$ denote the cardinality of some part; by forgetting all points not in this part, there is a section $s^{\prime}: \operatorname{Mod}_{n}\left(S^{2}\right) \rightarrow$ $\operatorname{Mod}_{n, m^{\prime}}\left(S^{2}\right)$. As $m$ is not divisible by $n(n-1)(n-2)$, any nontrivial partition of an $m$-element set necessarily has some part of cardinality $m^{\prime}<m$ not divisible by $n(n-1)(n-2)$. Such $m^{\prime}$ contradicts the minimality of $m$.

\subsection{Fixed points of torsion elements}

The essential distinction between the case $m \equiv 0 \bmod n(n-1)(n-2)$, where sections of $\operatorname{Conf}_{n, m}\left(S^{2}\right) \rightarrow \operatorname{Conf}_{n}\left(S^{2}\right)$ exist, and $m \neq 00 \bmod n(n-1)(n-2)$, where they do not, turns out to be the fact, recorded in Lemma 6.3 below, that in the latter cases, there always exists some torsion element $\alpha$ that fixes at least one new point.

Lemma 6.3 Suppose $m \not \equiv 0 \bmod n(n-1)(n-2)$, and that a section $s: \operatorname{Mod}_{n}\left(S^{2}\right) \rightarrow$ $\operatorname{Mod}_{n, m}\left(S^{2}\right)$ exists. Then at least one of $\alpha \in\left\{\alpha_{0}, \alpha_{1}\right\} \subset \operatorname{Mod}_{n}\left(S^{2}\right)$ has the property that $s(\alpha)$ fixes some new point $A$.

Proof We first claim that if a section exists, necessarily $m \equiv 0 \bmod (n-2)$. To see this, we study powers $\alpha_{2}^{k} \in \operatorname{Mod}_{n}\left(S^{2}\right)$ for $1 \leq k<n-2$. Such elements fix two old points, and hence $s\left(\alpha_{2}^{k}\right)$ also fixes these points. By Remark 5.3, $s\left(\alpha_{2}^{k}\right)$ has no further fixed points. This implies that the set of $m$ new points decomposes as a union of $s\left(\alpha_{2}\right)$-orbits, each of cardinality exactly $n-2$ (if $s\left(\alpha_{2}\right)$ did not act freely on the set of new points, some $s\left(\alpha_{2}^{k}\right)$ would fix some new point).

It follows that if $m$ is not divisible by $n(n-1)(n-2)$, then $m$ is not divisible by at least one of $n$ or $n-1$. If $m \equiv k(\bmod n)$ for some integer $1 \leq k<n$, then the action of $s\left(\alpha_{0}\right)$ on the set of new points has $k>0$ fixed points. Similar reasoning shows that $s\left(\alpha_{1}\right)$ has a new fixed point whenever $m \not \equiv 0 \bmod (n-1)$.

\subsection{Canonical reduction systems and trees}

We come now to the key object of interest. We will study the set

$$
\mathscr{C}:=\operatorname{CRS}\left(s\left(\sigma_{1}\right)\right) .
$$

The structure of $\mathscr{C}$ is best encoded as a graph. 
Definition 6.4 The graph $\mathscr{T}$ has vertices in bijection with the components of $S_{\mathscr{C}}^{2}$, and edges in bijection with elements of $\mathscr{C}$. An edge $c \in \mathscr{C}$ joins the components $S_{1}, S_{2} \subset S_{\mathscr{C}}^{2}$ for which $c$ is a boundary component of both $S_{1}$ and $S_{2}$.

Lemma 6.5 The graph $\mathscr{T}$ is a tree.

Proof It is clear from the construction that $\mathscr{T}$ is connected. Let $V$ and $E$ denote the number of vertices and edges of $\mathscr{T}$, respectively. As $\mathscr{T}$ is connected, it follows that $\mathscr{T}$ is a tree if and only if the Euler characteristic satisfies

$$
\chi(\mathscr{T})=V-E=1 .
$$

Enumerate the components of $S_{\mathscr{C}}^{2}$ as $S_{1}, \ldots, S_{V}$. A component $S_{i}$ of $S_{\mathscr{C}}^{2}$ has Euler characteristic $2-b_{i}$, where $b_{i}$ is the number of boundary components of $S_{i}$, ie the number of edges of $\mathscr{T}$ incident to $S_{i}$. Since each pair $S_{i}$ and $S_{j}$ of components of $S_{\mathscr{C}}^{2}$ meet in $S^{2}$ along a union of circles (each of Euler characteristic zero), the cut-and-paste formula

$$
\chi(A \cup B)=\chi(A)+\chi(B)-\chi(A \cap B)
$$

for the Euler characteristic gives the following expression for $\chi\left(S^{2}\right)$ :

$$
2=\chi\left(S^{2}\right)=\sum_{i=1}^{V}\left(2-b_{i}\right)=2 V-\sum_{i=1}^{V} b_{i}=2 V-2 E .
$$

The result follows.

\section{Proof of Theorem A: overview}

As we have already remarked, our standing assumption is that $n \geq 6$ and $m$ is the minimal integer not divisible by $n(n-1)(n-2)$ for which a section $s: \operatorname{Mod}_{n}\left(S^{2}\right) \rightarrow$ $\operatorname{Mod}_{n, m}\left(S^{2}\right)$ exists; Lemma 6.2 implies that $\Gamma$ acts transitively on the set of new points. Our strategy will be to derive a contradiction to the transitivity assumption, or else to show that $\Gamma$ is reducible: there exists a nonempty set $\mathscr{R}$ of disjoint essential curves in $S_{n+m}^{2}$ satisfying $\Gamma(\mathscr{R})=\mathscr{R}$. By Lemmas 7.1 and 7.2 below, this will also produce a contradiction. Lemmas 7.1 and 7.2 are established in Section 8. At this juncture we remind the reader of the standard convention that isotopies are required to pointwise fix all marked points; thus when $S^{2}$ is equipped with $n$ old points and $m$ new points, it is sensible to study which isotopy classes of subsurfaces contain which points. 
Lemma 7.1 Fix $n \geq 3$, and let $s: \operatorname{Mod}_{n}\left(S^{2}\right) \rightarrow \operatorname{Mod}_{n, m}\left(S^{2}\right)$ be a section of the map $p: \operatorname{Mod}_{n, m}\left(S^{2}\right) \rightarrow \operatorname{Mod}_{n}\left(S^{2}\right)$. Suppose that $\Gamma$ acts transitively on the set of new points, and that there is a $\Gamma$-invariant subsurface $S \subset S^{2}$ that contains at least one old point. Then either $m$ is divisible by $n(n-1)(n-2)$, or else there is some $m^{\prime}<m$ with $m^{\prime}$ not divisible by $n(n-1)(n-2)$ and a section $s^{\prime}: \operatorname{Mod}_{n}\left(S^{2}\right) \rightarrow \operatorname{Mod}_{n, m^{\prime}}\left(S^{2}\right)$.

Lemma 7.2 Fix $n \geq 3$, and let $s: \operatorname{Mod}_{n}\left(S^{2}\right) \rightarrow \operatorname{Mod}_{n, m}\left(S^{2}\right)$ be a section of the map $p: \operatorname{Mod}_{n, m}\left(S^{2}\right) \rightarrow \operatorname{Mod}_{n}\left(S^{2}\right)$. Suppose that $\Gamma$ acts transitively on the set of new points, and that there is a $\Gamma$-invariant set $\left\{S_{1}, \ldots, S_{n}\right\}$ of subsurfaces, each with a single boundary component $c_{i}$, such that $x_{i} \in S_{i}$ for $i=1, \ldots, n$. Then $m$ is divisible by $n(n-1)(n-2)$.

The argument proceeds by studying some distinguished components of $S_{\mathscr{C}}^{2}$. For $i=3, \ldots, n$, let $S_{i}$ be the component of $S_{\mathscr{C}}^{2}$ that contains the old point $x_{i}$. The $S_{i}$ are not necessarily pairwise distinct. To get a better understanding of the set $\left\{S_{i}\right\}$, we make the following observations. By Lemma 5.4, if $g \in \operatorname{Mod}_{n}\left(S^{2}\right)$ commutes with $\sigma_{1}$, then $s(g)$ induces a permutation of $\mathscr{C}$ and hence an automorphism $g_{*}$ of the tree $\mathscr{T}$. It will be useful to view this automorphism in geometric terms. Let $\delta$ be the metric on $\mathscr{T}$ in which all edges have length 1 .

Proposition 7.3 The induced automorphism $g_{*}$ of the tree $\mathscr{T}$ is an isometry of the metric space $(\mathscr{T}, \delta)$.

Proof Graph automorphisms are simplicial and hence distance-nonincreasing with respect to $\delta$; as this applies to both $g_{*}^{ \pm 1}$ it follows that $g_{*}$ is an isometry.

Moreover, the following lemma shows that there is a large supply of such elements $g$ for which the behavior on the set of old points is prescribed. The proof is elementary and is omitted.

Lemma 7.4 For any pair of distinct old points $x_{i}$ and $x_{j}$ with $i, j \geq 3$, there exists an element $g \in \operatorname{Mod}_{n}\left(S^{2}\right)$ such that $g$ commutes with $\sigma_{1}$ and such that $g\left(x_{i}\right)=x_{3}$ and $g\left(x_{j}\right)=x_{4}$.

For any $g$ as in Lemma 7.4, since $s(g)$ permutes the components of $S_{\mathscr{C}}^{2}$ and $g\left(x_{i}\right)=x_{3}$, it follows that $g_{*}\left(S_{i}\right)=S_{3}$. Similarly $g_{*}\left(S_{j}\right)=S_{4}$. Thus, in the metric graph $(\mathscr{T}, \delta)$,

$$
\delta\left(S_{i}, S_{j}\right)=\delta\left(g_{*}\left(S_{i}\right), g_{*}\left(S_{j}\right)\right)=\delta\left(S_{3}, S_{4}\right) .
$$


Therefore $\delta\left(S_{i}, S_{j}\right)=d$, a constant which does not depend on $i$ or $j$. There are two possibilities: either

Case A $d=0$, so that $S:=S_{3}=\cdots=S_{n}$, or

Case B $d>0$, so that each $S_{3}, \ldots, S_{n}$ is a distinct subsurface of $S_{\mathscr{C}}^{2}$.

To analyze Case A, we appeal to the theory of canonical reduction systems. Since $s\left(\sigma_{1}\right)$ fixes a point $x_{3} \in S$, it follows that $s\left(\sigma_{1}\right)$ fixes the component $S$. Lemma 5.2 then implies that the restriction of $s\left(\sigma_{1}\right)$ to $S$ is either pseudo-Anosov or else periodic. We handle each possibility in turn, as Cases A.1 and A.2, respectively. Case A.1 is resolved by showing that $S$ is necessarily $\Gamma$-invariant; this contradicts Lemma 7.1.

The analysis of Case A.2, where $s\left(\sigma_{1}\right)$ is assumed to be periodic, requires a further division into subcases. Lemma 6.3 guarantees the existence of torsion elements of $\Gamma$ that fix at least one new point $A$. Case A.2 further subdivides, depending on whether $A$ is contained in $S$ or not. In Case A.2.a, where $A \in S$, we will show that either $A$ is a global fixed point, contradicting transitivity, or else that there is a nontrivial torsion element with 3 fixed points, contradicting Remark 5.3. In the alternative Case A.2.b, we will produce an essential $\Gamma$-invariant curve, contradicting Lemma 7.1.

The ultimate aim in Case B is to show that $\Gamma$ is reducible. In Lemma 10.1, we produce a collection $c_{3}, \ldots, c_{n}$ of distinguished boundary components of $S_{3}, \ldots, S_{n}$. After analyzing how $s\left(\alpha_{0}\right)$ acts on this set in Lemma 10.2, we are able to define two further curves $c_{1}$ and $c_{2}$. We then show in Lemma 10.4 that the set of curves $\left\{c_{1}, \ldots, c_{n}\right\}$ is $\Gamma$-invariant, leading to a contradiction with Lemma 7.2.

\section{Proof of Theorem A: the reducible case}

In this section we treat the situation where $\Gamma:=s\left(\operatorname{Mod}_{n}\left(S^{2}\right)\right)$ is reducible. The objective is to prove Lemmas 7.1 and 7.2.

Proof of Lemma 7.1 A first observation is that $S$ contains all old points. Indeed, if $x_{i} \in S$, then for any $j=1, \ldots, n$, there exists $\phi_{j} \in \Gamma$ for which $\phi_{j}\left(x_{i}\right)=x_{j}$. As $x_{i} \in S$ and $S$ is $\Gamma$-invariant, it follows that $x_{j} \in S$ as well.

By hypothesis, $s$ is valued in the subgroup $\operatorname{Mod}_{n, m}\left(S^{2}, S\right)$ of mapping classes that preserve the subsurface $S$. There is a restriction map

$$
r: \operatorname{Mod}_{n, m}\left(S^{2}, S\right) \rightarrow \operatorname{Mod}_{n}(S) \cong \operatorname{Mod}_{n, m^{\prime}+m^{\prime \prime}}\left(S^{2}\right),
$$


where $m^{\prime}$ is the number of boundary components of $S$, and $m^{\prime \prime}$ is the number of new points contained in $S$. Setting $s^{\prime}:=r \circ s$, we obtain a new homomorphism

$$
s^{\prime}: \operatorname{Mod}_{n}\left(S^{2}\right) \rightarrow \operatorname{Mod}_{n, m^{\prime}+m^{\prime \prime}}\left(S^{2}\right) .
$$

We claim that $m^{\prime \prime}=0$, that $m^{\prime}<m$, and that if $m^{\prime}$ is divisible by $n(n-1)(n-2)$, then $m$ is as well. To see these claims, observe that since $S$ contains all of the old points, each component of $S^{2} \backslash S$ contains only new points. Since each boundary component of $S$ is essential in $S_{m+n}^{2}$, there must be at least two new points contained in each component of $S^{2} \backslash S$; this shows $m^{\prime}<m$. By hypothesis, $\Gamma$ acts transitively on the set of new points. Since $S$ is $\Gamma$-invariant, any new points contained in $S$ cannot be exchanged with new points off of $S$, and so $m^{\prime \prime}=0$, as claimed. Moreover, $\Gamma$ must act transitively on the set of components of $S^{2} \backslash S$. Letting $p$ denote the number of new points contained in each component, we see that $m=m^{\prime} p$. Thus if $m^{\prime}$ is divisible by $n(n-1)(n-2)$, so is $m$.

To establish Lemma 7.1, it now suffices to show that $s^{\prime}$ is a section of the forgetful $\operatorname{map} p^{\prime}: \operatorname{Mod}_{n, m^{\prime}}\left(S^{2}\right) \rightarrow \operatorname{Mod}_{n}\left(S^{2}\right)$. Recall that $s: \operatorname{Mod}_{n}\left(S^{2}\right) \rightarrow \operatorname{Mod}_{n, m}\left(S^{2}\right)$ is a section of the forgetful map $p: \operatorname{Mod}_{n, m}\left(S^{2}\right) \rightarrow \operatorname{Mod}_{n}\left(S^{2}\right)$. The claim now follows from $s^{\prime}=r \circ s$ and the factorizations

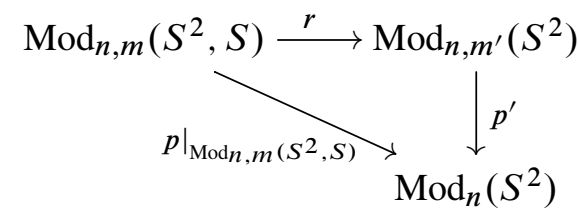

Proof of Lemma 7.2 We claim that all new points are contained inside the set

$$
\bigcup_{i=1}^{n} S_{i} \text {. }
$$

Certainly there must exist some new point in each $S_{i}$, as otherwise $c_{i}$ would be inessential. Since $\Gamma$ acts transitively on the set of new points and the set $\bigcup S_{i}$ is $\Gamma$-invariant, the claim follows.

To conclude the argument, we count the number of new points. As $\Gamma$ permutes the subsurfaces $S_{i}$, each contains the same number $m^{\prime}$ of new points. For any $i=1, \ldots, n$, there is a conjugate $\alpha_{2, i}$ of $\alpha_{2}$ that fixes the point $x_{i}$. It follows that $S_{i}$ is $s\left(\alpha_{2, i}\right)-$ invariant, and hence the set of new points contained in $S_{i}$ decomposes as a union of orbits of $s\left(\alpha_{2, i}\right)$. By Remark 2.3, each orbit contains $n-2$ points, so that $(n-2) \mid m^{\prime}$. 
Likewise, let $\alpha_{1, i}$ be a conjugate of $\alpha_{1}$ that fixes $x_{i}$. Then $s\left(\alpha_{1, i}\right)$ also fixes $S_{i}$ and so decomposes the new points in $S_{i}$ into a union of orbits. By Remark 2.3, each orbit contains $n-1$ points, so that also $(n-1) \mid m^{\prime}$. We conclude that $(n-1)(n-2) \mid m^{\prime}$, and as $m=n m^{\prime}$, Lemma 7.2 follows.

\section{Proof of Theorem A: Case A}

The assumption in Case A is that $S:=S_{3}=S_{4}=\cdots=S_{n}$. As discussed in the overview given in Section 7, Case A divides into two subcases.

Case A.1 $s\left(\sigma_{1}\right)$ is pseudo-Anosov on $S$.

By Lemma 2.4, $\alpha_{0}^{2} \sigma_{1} \alpha_{0}^{-2}=\sigma_{3}$. Therefore,

$$
\operatorname{CRS}\left(s\left(\sigma_{3}\right)\right)=\operatorname{CRS}\left(s\left(\alpha_{0}^{2} \sigma_{1} \alpha_{0}^{-2}\right)\right)=s\left(\alpha_{0}^{2}\right) \cdot \operatorname{CRS}\left(s\left(\sigma_{1}\right)\right)=s\left(\alpha_{0}^{2}\right) \cdot \mathscr{C} .
$$

Since $\sigma_{1}$ and $\sigma_{3}$ commute, Lemma 5.5 implies that any pair of curves $c \in \operatorname{CRS}\left(s\left(\sigma_{3}\right)\right)$ and $d \in \operatorname{CRS}\left(s\left(\sigma_{1}\right)\right)=\mathscr{C}$ are disjoint or else equal. Let $\partial S \subset \mathscr{C}$ denote the set of boundary components of $S$. Then every element of $s\left(\alpha_{0}^{2}\right)(\partial S)$ is disjoint from the elements of $\partial S$, or else is also an element of $\partial S$. Each component of $s\left(\alpha_{0}^{2}\right)(\partial S)$ is an element of $\operatorname{CRS}\left(s\left(\sigma_{3}\right)\right)$, and since $s\left(\sigma_{1}\right)$ and $s\left(\sigma_{3}\right)$ commute, some power of $s\left(\sigma_{1}\right)$ must preserve each such component. Since $s\left(\sigma_{1}\right)$ is pseudo-Anosov on $S$, no power of $s\left(\sigma_{1}\right)$ fixes a curve in $S$. Therefore none of the elements of $s\left(\alpha_{0}^{2}\right)(\partial S)$ are contained in the interior of $S$.

On the other hand, $S$ and $s\left(\alpha_{0}^{2}\right)(S)$ contain the same number of punctures and each contains the puncture $x_{5}$. Lemma 5.6 then implies that $\alpha_{0}^{2}(S)=S$. By the same reasoning, $\alpha_{0}^{3}(S)=S$, and it follows that $\alpha_{0}(S)=S$. By Lemma 2.5, the entire group $\Gamma$ preserves the component $S$. Thus the hypotheses of Lemma 7.1 are satisfied, leading to a contradiction with the minimality assumption on $m$.

Case A.2 $s\left(\sigma_{1}\right)$ is periodic on $S$.

We first claim that in fact $s\left(\sigma_{1}\right)$ is the identity on $S$. This follows from the fact that $\sigma_{1}$ fixes at least the three old points $x_{3}, x_{4}, x_{5} \in S$, in combination with Remark 5.3.

As in Lemma 6.3, let $\alpha$ be whichever of $\alpha_{0}$ and $\alpha_{1}$ fixes some new point $A$. There are two possibilities: 


\section{Case A.2.a $A \in S$.}

Since $s\left(\sigma_{1}\right)$ acts by the identity on $S$, necessarily $s\left(\sigma_{1}\right)(A)=A$. Since $s(\alpha)(A)=A$ by construction, it follows that the subgroup $G \leqslant \operatorname{Mod}_{n, m}\left(S^{2}\right)$ generated by $s\left(\sigma_{1}\right)$ and $s(\alpha)$ fixes $A$. If $\alpha=\alpha_{0}$, Lemma 2.5 implies that $G=\Gamma$. But then $\Gamma$ does not act transitively on the set of new points, in contradiction with Lemma 6.2.

If $\alpha=\alpha_{1}$, Lemma 2.6 implies that $G=s\left(\operatorname{Mod}_{n-1,1}\left(S^{2}\right)\right)$. Thus $s$ restricts to give an injective homomorphism

$$
s: \operatorname{Mod}_{n-1,1}\left(S^{2}\right) \rightarrow \operatorname{Mod}_{n-1,1, m-1,1}\left(S^{2}\right),
$$

where $\operatorname{Mod}_{n-1,1, m-1,1}\left(S^{2}\right)<\operatorname{Mod}_{n, m}$ is the subgroup consisting of elements that fix $x_{n}$ and $A$. The element $\alpha_{2} \in \operatorname{Mod}_{n}\left(S^{2}\right)$ is contained in $\operatorname{Mod}_{n-1,1}\left(S^{2}\right)$ and is torsion of order $n-2$ with two fixed points $x_{n-1}$ and $x_{n}$, both old. Thus $s\left(\alpha_{2}\right)$ must also be torsion of order $n-2$ with two fixed old points. By Remark 5.3, $s\left(\alpha_{2}\right)$ cannot have any further fixed points, but by definition every element of $\operatorname{Mod}_{n-1,1, m-1,1}\left(S^{2}\right)$ fixes the new point $A$, a contradiction.

Case A.2.b $A \notin S$.

In this case, there exists a curve in $c \in \partial S$ separating $x_{3}, \ldots, x_{n}$ from $A$. Such a $c$ is necessarily $s\left(\sigma_{1}\right)$-invariant, since $s\left(\sigma_{1}\right)$ acts as the identity on $S$. We claim that $c$ must also be $s(\alpha)$-invariant, and must moreover preserve the subsurfaces on either side of $c$.

Proof of claim Since $n \geq 6$, Lemma 2.4 implies that $\alpha^{2} \sigma_{1} \alpha^{-2}=\sigma_{3}$, and thus $s\left(\alpha^{2}\right)(c)$ belongs to $\operatorname{CRS}\left(s\left(\sigma_{3}\right)\right)$. It follows that $s\left(\alpha^{2}\right)(c)$ is either disjoint from $c$ or else $s\left(\alpha^{2}\right)(c)=c$. We will see that $s\left(\alpha^{2}\right)(c)=c$ must hold. Let $S_{A} \subset S^{2}$ denote the subsurface bounded by $c$ that contains $A$.

We claim that the pair of surfaces $S_{A}$ and $s\left(\alpha^{2}\right)\left(S_{A}\right)$ satisfy the hypotheses of Lemma 5.6. Each surface has a single boundary component, respectively $c$ and $s\left(\alpha^{2}\right)(c)$, and we have already established that $c$ and $s\left(\alpha^{2}\right)(c)$ are either disjoint or equal. Each surface contains the point $A$, and as they are conjugate within $\Gamma$, each contains the same number of punctures.

It remains to show that $s\left(\alpha^{2}\right)(c)$ is not contained in the interior of $S_{A}$. If this is the case, then $s\left(\alpha^{2}\right)(c)$ encloses a strict subset of the punctures contained in $S_{A}$. The curve $c$ induces a partition $P=P_{1} \cup P_{2}$ of the set of punctures, and likewise $s\left(\alpha^{2}\right)(c)$ induces the conjugate partition $s\left(\alpha^{2}\right)(P)$. Without loss of generality, assume that $P_{1}$ 
corresponds to the punctures in $S_{A}$ and hence contains $A$, so that $P_{2}$ contains the points $x_{3}, \ldots, x_{n}$. Since $s\left(\alpha^{2}\right)(c)$ encloses a strict subset of the punctures contained in $S_{A}$, one of the parts of $s\left(\alpha^{2}\right)(P)$ must be a strict subset of $P_{1}$. This part cannot be $s\left(\alpha^{2}\right)\left(P_{1}\right)$, since $s\left(\alpha^{2}\right)\left(P_{1}\right)$ has the same cardinality as $P_{1}$. But this part cannot be $s\left(\alpha^{2}\right)\left(P_{2}\right)$ either, since $P_{2}$ contains $x_{3}$ and hence $s\left(\alpha^{2}\right)\left(P_{2}\right)$ contains $x_{5} \in P_{2}$.

By Lemma 5.6, we have $s(\alpha)^{2}\left(S_{A}\right)=S_{A}$. As $n \geq 6$, also $\alpha^{3} \sigma_{1} \alpha^{-3}=\sigma_{4}$. The same argument then shows that $s\left(\alpha^{3}\right)\left(S_{A}\right)=S_{A}$, and hence $s(\alpha)\left(S_{A}\right)=S_{A}$. The claim follows.

We have shown that $s\left(\sigma_{1}\right)$ and $s(\alpha)$ both fix $c$ as well as the subsurfaces on either side of $c$. Let $S$ be the side containing the points $x_{3}, \ldots, x_{n}$. In the case $\alpha=\alpha_{0}$, necessarily $S$ is globally invariant, in contradiction with Lemma 7.1. If $\alpha=\alpha_{1}$, then we have shown that the image of the subgroup $\operatorname{Mod}_{n-1,1}\left(S^{2}\right)=\left\langle\sigma_{1}, \alpha_{1}\right\rangle$ under $s$ is contained in the subgroup $\operatorname{Mod}_{n-1,1, m}\left(S^{2}, S\right)$ of mapping classes fixing $S$. Composing with the map $r: \operatorname{Mod}_{n-1,1, m}\left(S^{2}, S\right) \rightarrow \operatorname{Mod}_{n-1,1, m^{\prime}, 1}\left(S^{2}\right)$ obtained by restriction to $S$, we can now conclude the argument exactly as in the preceding Case A.2.a.

\section{Proof of Theorem A: Case B}

The assumption in Case B is that the subsurfaces $S_{3}, \ldots, S_{n}$ are all distinct. This case follows by an analysis of the boundary components of the subsurfaces $S_{i}$. A first observation is that the (necessarily disjoint) subsurfaces $S_{i}$ are all conjugate within $\Gamma$ : for any $i \geq 3$, there is some $g \in \operatorname{Mod}_{n}\left(S^{2}\right)$ commuting with $\sigma_{1}$ and taking $x_{3}$ to $x_{i}$. Then $s(g)\left(S_{3}\right)=S_{i}$.

Lemma 10.1 For each $i=3, \ldots, n$, there is a unique component $c_{i}$ of $\partial S_{i}$ that separates $S_{i}$ from $p>\frac{1}{2}(n+m)$ punctures.

Proof Since the subsurfaces $S_{i}$ are all conjugate within $\Gamma$, it suffices to consider only $S_{3}$. Certainly if $c_{3}$ exists it must be unique. To see that it exists, denote the boundary components of $S_{3}$ by $d_{1}, \ldots, d_{k}$. For $1 \leq i \leq k$, let $D_{i}$ denote the disk bounded by $d_{i}$ not containing $S_{3}$, and let $n_{i}$ denote the number of punctures in $D_{i}$. Without loss of generality, assume that $S_{3}$ is separated from $S_{4}=s\left(\sigma_{3}\right)\left(S_{3}\right)$ by $d_{1}$. Reversing the roles of $S_{3}$ and $S_{4}$, we find that $S_{4}$ is separated from $S_{3}$ by some other element $s\left(\sigma_{3}\right)\left(d_{i}\right) \in \mathscr{C}$ for some $1 \leq i \leq k$. We claim that $i=1$, since if $i>1$, then 
there is a strict containment $D_{i} \subset S^{2}-D_{1} \subset s\left(\sigma_{3}\right)\left(D_{i}\right)$, an absurdity. It follows that there is a containment

$$
D_{2} \cup \cdots \cup D_{k} \cup S_{3} \subset s\left(\sigma_{3}\right)\left(D_{1}\right),
$$

and hence, letting $n_{0}$ denote the number of punctures contained in $S_{3}$ itself,

$$
n_{1} \geq n_{2}+\cdots+n_{k}+n_{0}
$$

On the other hand, $\sum_{i=0}^{k} n_{i}=n+m$, from which the inequality $n_{1} \geq \frac{1}{2}(n+m)$ follows. Moreover, this inequality must be strict, since otherwise the disks $D_{1}$ and $s\left(\sigma_{3}\right)\left(D_{1}\right)$ would be disjoint because their boundaries are disjoint, they both contain half of the points and they contain different points. For the same reason, $s\left(\sigma_{3}\right)\left(D_{1}\right)$ and $s\left(\sigma_{4} \sigma_{3}\right)\left(D_{1}\right)$ would be mutually disjoint and each would contain $\frac{1}{2}(n+m)$ points. This is absurd. Taking $c_{3}:=d_{1}$, the result follows.

Lemma 10.2 $s\left(\alpha_{0}^{2}\right)\left(c_{3}\right)=c_{5}$ and $s\left(\alpha_{0}^{3}\right)\left(c_{3}\right)=c_{6}$.

Proof Define the inside of each $c_{i}$ to be the component $\operatorname{Int}\left(c_{i}\right)$ of $S_{\mathscr{C}}^{2}$ that contains $x_{i}$, and define the outside as the other component. Each $\operatorname{Int}\left(c_{i}\right)$ contains $q$ punctures, with $q<\frac{1}{2}(n+m)$ by Lemma 10.1. Define $c_{5}^{\prime}:=s\left(\alpha_{0}^{2}\right)\left(c_{3}\right)$. We again define the inside of $c_{5}^{\prime}$ as the component $\operatorname{Int}\left(c_{5}^{\prime}\right)$ containing $x_{5}$, and the outside as the other component.

We claim that $\operatorname{Int}\left(c_{5}\right)$ and $\operatorname{Int}\left(c_{5}^{\prime}\right)$ satisfy the hypotheses of Lemma 5.6. As $c_{5} \in \mathscr{C}$ and $c_{5}^{\prime} \in s\left(\alpha_{0}\right)^{2} \mathscr{C}=\operatorname{CRS}\left(s\left(\sigma_{3}\right)\right)$, we have that $c_{5}$ and $c_{5}^{\prime}$ are either disjoint or equal. By definition, each contains $x_{5}$. The curves $c_{3}$ and $c_{5}$ contain the same number of punctures on their interiors, hence the same is true of $c_{5}$ and $c_{5}^{\prime}$. It remains to be seen that $c_{5}^{\prime}$ is not contained in the interior of $\operatorname{Int}\left(c_{5}\right)$. If this is the case, then either the inside or the outside of $c_{5}^{\prime}$ contains strictly fewer than $q$ punctures. But as the inside of $c_{5}^{\prime}$ contains $q$ punctures and the outside contains $n+m-q>q$ punctures, this cannot be the case.

Applying Lemma 5.6, it follows that $c_{5}=c_{5}^{\prime}=s\left(\alpha_{0}\right)^{2}\left(c_{3}\right)$, as claimed. Similar arguments establish the other claim.

Define the curves $c_{1}=s\left(\alpha_{0}^{-2}\right)\left(c_{3}\right)$ and $c_{2}=s\left(\alpha_{0}^{-2}\right)\left(c_{4}\right)$.

Lemma 10.3 The curves $c_{1}, \ldots, c_{n}$ are pairwise distinct and disjoint. 
Proof The curves $c_{3}, \ldots, c_{n}$ are distinct and disjoint since they are all elements of $\mathscr{C}$ and each $c_{i}$ is distinguished by the property that it contains $x_{i}$ on its inside. The curves $c_{1}$ and $c_{2}$ are elements of $s\left(\alpha_{0}^{-2}\right) \mathscr{C}=\operatorname{CRS}\left(\sigma_{n-1}\right)$, and hence either disjoint from or equal to any element of $\mathscr{C}$. But $c_{1}$ and $c_{2}$ are uniquely characterized by the property of respectively containing $x_{1}$ and $x_{2}$ in their interiors, and the claim follows.

Lemma 10.4 The set $\left\{c_{1}, c_{2}, \ldots, c_{n}\right\}$ is invariant under $\Gamma$.

Proof By Lemma 2.5, $\Gamma$ is generated by the set $\left\{s\left(\sigma_{1}\right), s\left(\alpha_{0}\right)\right\}$. Thus it suffices to show that these two elements both preserve $\left\{c_{1}, \ldots, c_{n}\right\}$. For $i=3, \ldots, n$, the element $s\left(\sigma_{1}\right)$ preserves each $S_{i}$, and hence also preserves the distinguished boundary component $c_{i}$. We claim that $s\left(\sigma_{1}\right)\left(c_{1}\right)=c_{2}$ and that $s\left(\sigma_{1}\right)\left(c_{2}\right)=c_{1}$.

To see this, observe that $s\left(\sigma_{3}\right)\left(c_{3}\right)=c_{4}$. Thus $s\left(\alpha_{0}^{2} \sigma_{1} \alpha_{0}^{-2}\right)\left(c_{3}\right)=c_{4}$, and so

$$
s\left(\alpha_{0}^{2}\right) s\left(\sigma_{1}\right)\left(s\left(\alpha_{0}^{-2}\right)\left(c_{3}\right)\right)=c_{4}=s\left(\alpha_{0}^{2}\right)\left(c_{2}\right) .
$$

It follows that $s\left(\sigma_{1}\right)\left(s\left(\alpha_{0}^{-2}\right)\left(c_{3}\right)\right)=s\left(\sigma_{1}\right)\left(c_{1}\right)=c_{2}$, as claimed. As also $s\left(\sigma_{3}\right)\left(c_{4}\right)=c_{3}$, the same reasoning shows that $s\left(\sigma_{1}\right)\left(c_{2}\right)=c_{1}$.

It remains to see that the set $\left\{c_{1}, \ldots, c_{n}\right\}$ is $\alpha_{0}$-invariant. We claim that $\alpha_{0}\left(c_{i}\right)=c_{i+1}$, interpreting subscripts mod $n$. It follows directly from Lemma 10.2 that $s\left(\alpha_{0}\right)\left(c_{5}\right)=c_{6}$. As $\alpha_{0} \sigma_{4}=\sigma_{5} \alpha_{0}$,

$$
s\left(\alpha_{0}\right)\left(c_{4}\right)=s\left(\alpha_{0} \sigma_{4}\right)\left(c_{5}\right)=s\left(\sigma_{5}\right) s\left(\alpha_{0}\right)\left(c_{5}\right)=s\left(\sigma_{5}\right)\left(c_{6}\right)=c_{5},
$$

since $s\left(\sigma_{i}\right)\left(c_{i+1}\right)=c_{i}$ for $i=3, \ldots, n-1$. Similar arguments show that $s\left(\alpha_{0}\right)\left(c_{3}\right)=c_{4}$, and $s\left(\alpha_{0}\right)\left(c_{i}\right)=c_{i+1}$ for $i=6, \ldots, n-1$.

There are three remaining claims to establish:

$$
s\left(\alpha_{0}\right)\left(c_{1}\right)=c_{2}, \quad s\left(\alpha_{0}\right)\left(c_{2}\right)=c_{3}, \quad s\left(\alpha_{0}\right)\left(c_{n}\right)=c_{1} .
$$

Since $c_{2}:=s\left(\alpha_{0}^{-2}\right)\left(c_{4}\right)$, the equality $s\left(\alpha_{0}\right)\left(c_{2}\right)=c_{3}$ follows from the above. Then the equality $s\left(\alpha_{0}\right)\left(c_{1}\right)=c_{2}$ follows by the same logic. Lastly, as $\alpha_{0}^{n}=\mathrm{id}$,

$$
s\left(\alpha_{0}\right)\left(c_{n}\right)=s\left(\alpha_{0}^{1-n}\right)\left(c_{n}\right)=c_{1}
$$

by what we have shown before.

Lemmas 10.4 and 7.2 combine to show that $m$ must be divisible by $n(n-1)(n-2)$, contrary to assumption. Case B, and hence Theorem A, follow. 


\section{References}

[1] E Artin, Theory of braids, Ann. of Math. 48 (1947) 101-126 MR

[2] J S Birman, A Lubotzky, J McCarthy, Abelian and solvable subgroups of the mapping class groups, Duke Math. J. 50 (1983) 1107-1120 MR

[3] L Chen, Section problems for configuration spaces of surfaces, J. Topol. Anal. (online publication July 2019)

[4] W Chen, Obstructions to choosing distinct points on cubic plane curves, Adv. Math. 340 (2018) 211-220 MR

[5] F R Cohen, J Wu, On braid groups and homotopy groups, from "Groups, homotopy and configuration spaces" (N Iwase, T Kohno, R Levi, D Tamaki, J Wu, editors), Geom. Topol. Monogr. 13, Geom. Topol. Publ., Coventry (2008) 169-193 MR

[6] C J Earle, I Kra, On holomorphic mappings between Teichmüller spaces, from "Contributions to analysis" (L V Ahlfors, I Kra, B Maskit, L Nirenberg, editors), Academic, New York (1974) 107-124 MR

[7] C J Earle, I Kra, On sections of some holomorphic families of closed Riemann surfaces, Acta Math. 137 (1976) 49-79 MR

[8] B Farb, D Margalit, A primer on mapping class groups, Princeton Math. Series 49, Princeton Univ. Press (2012) MR

[9] E Formanek, Braid group representations of low degree, Proc. Lond. Math. Soc. 73 (1996) 279-322 MR

[10] D L Gonçalves, J Guaschi, The braid group $B_{n, m}\left(\mathbb{S}^{2}\right)$ and a generalisation of the Fadell-Neuwirth short exact sequence, J. Knot Theory Ramif. 14 (2005) 375-403 MR

[11] J Guaschi, D Juan-Pineda, A survey of surface braid groups and the lower algebraic $K$-theory of their group rings, from "Handbook of group actions, II" ( $\mathrm{L} \mathrm{Ji}, \mathrm{A}$ Papadopoulos, S-T Yau, editors), Adv. Lect. Math. 32, International, Somerville, MA (2015) 23-75 MR

[12] R Hartshorne, Algebraic geometry, Grad. Texts in Math. 52, Springer (1977) MR

[13] J H Hubbard, Sur les sections analytiques de la courbe universelle de Teichmüller, Mem. Amer. Math. Soc. 166, Amer. Math. Soc., Providence, RI (1976) MR

[14] VY Lin, Algebraic functions, configuration spaces, Teichmüller spaces, and new holomorphically combinatorial invariants, Funktsional. Anal. i Prilozhen. 45 (2011) 55-78 MR In Russian; translated in Funct. Anal. Appl. 45 (2011), 204-224

[15] K Murasugi, Seifert fibre spaces and braid groups, Proc. Lond. Math. Soc. 44 (1982) 71-84 MR

[16] H Wenzl, Representations of braid groups and the quantum Yang-Baxter equation, Pacific J. Math. 145 (1990) 153-180 MR 
Department of Mathematics, Caltech

Pasadena, CA, United States

Department of Mathematics, Columbia University

New York, NY, United States

chenlei@caltech.edu, nks@math.columbia.edu

Received: 6 June 2019 Revised: 26 October 2019 